\title{
Supporting Information for Air-Induced anti-Markovnikov Addition of Secondary Phosphine Oxides and H-Phosphinates to Alkenes
}

Takayoshi Hirai and Li-Biao Han*

libiao-han@aist.go.jp

National Institute of Advanced Industrial Science and Technology, Tsukuba, Ibaraki 305-8565, Japan

\section{Contents}

S1-S4 Spectra and analytical data for new compounds

S5 - S68 Copies of NMR spectra for all products

\section{Spectra and analytical data for new compounds}

$\mathrm{Ph}(t-\mathrm{Bu})(\mathrm{O}) \mathrm{P} \frown n-\mathrm{C}_{8} \mathrm{H}_{17}$

(Table 2, run 2)

Colorless oil; ${ }^{1} \mathrm{H}$ NMR (499.1 MHz, $\mathrm{CDCl}_{3}$ ) $\delta$ 7.72-7.68 (m, 2H), 7.53-7.46 (m, 3H), 2.04-1.99 (m, 2H), 1.74-1.70 (m, 1H), 1.37-1.10 (m, 24H), 0.87 (t, $J=6.4 \mathrm{~Hz}, 3 \mathrm{H}) ;{ }^{13} \mathrm{C}$ NMR $(125.4 \mathrm{MHz}$, $\left.\mathrm{CDCl}_{3}\right) \delta 131.9\left(\mathrm{~d}, J_{\mathrm{P}-\mathrm{C}}=8.3 \mathrm{~Hz}\right), 131.3\left(\mathrm{~d}, J_{\mathrm{P}-\mathrm{C}}=2.1 \mathrm{~Hz}\right), 130.3\left(\mathrm{~d}, J_{\mathrm{P}-\mathrm{C}}=85.8 \mathrm{~Hz}\right), 128.2\left(\mathrm{~d}, J_{\mathrm{P}-\mathrm{C}}\right.$ $=10.3 \mathrm{~Hz}$ ), 32.7 (d, $\left.J_{\mathrm{P}-\mathrm{C}}=69.2 \mathrm{~Hz}\right), 31.9,31.3\left(\mathrm{~d}, J_{\mathrm{P}-\mathrm{C}}=13.4 \mathrm{~Hz}\right), 29.5\left(\mathrm{~d}, J_{\mathrm{P}-\mathrm{C}}=14.4 \mathrm{~Hz}\right), 29.2(\mathrm{~d}$, $\left.J_{\mathrm{P}-\mathrm{C}}=14.5 \mathrm{~Hz}\right), 24.6,23.8,22.9\left(\mathrm{~d}, J_{\mathrm{P}-\mathrm{C}}=66.2 \mathrm{~Hz}\right), 22.7,21.5\left(\mathrm{~d}, J_{\mathrm{P}-\mathrm{C}}=5.1 \mathrm{~Hz}\right), 14.1 ;{ }^{31} \mathrm{P} \mathrm{NMR}$ (201.95 MHz, $\left.\mathrm{CDCl}_{3}\right) \delta$ 49.7; HRMS $\left(\mathrm{M}^{+}\right)$calcd for $\mathrm{C}_{20} \mathrm{H}_{35} \mathrm{O}_{1} \mathrm{P}_{1}$ 322.2426, found 322.2415. Anal. Calcd for $\mathrm{C}_{20} \mathrm{H}_{35} \mathrm{O}_{1} \mathrm{P}_{1}$ : C, 74.49; H, 10.94. Found: C, 74.65; H, 10.55 .

$\mathrm{Ph}_{2}(\mathrm{O}) \mathrm{P} \leadsto\left(\mathrm{CH}_{2}\right)_{4} \mathrm{CN}$

Colorless oil; ${ }^{1} \mathrm{H}$ NMR (499.1 MHz, $\left.\mathrm{CDCl}_{3}\right) \delta$ 7.75-7.72 (m, 4H), 7.54-7.46 (m, 6H), 2.31-2.24 (m, 4H), 1.65-1.57 (m, 4H), 1.45-1.43 (m, 4H); ${ }^{13} \mathrm{C}$ NMR (125.4 MHz, $\left.\mathrm{CDCl}_{3}\right) \delta 133.0\left(\mathrm{~d}, J_{\mathrm{P}-\mathrm{C}}=\right.$ $98.2 \mathrm{~Hz}), 131.7$ (d, $\left.J_{\mathrm{P}-\mathrm{C}}=3.1 \mathrm{~Hz}\right), 130.7\left(\mathrm{~d}, J_{\mathrm{P}-\mathrm{C}}=9.3 \mathrm{~Hz}\right), 128.7\left(\mathrm{~d}, J_{\mathrm{P}-\mathrm{C}}=12.3 \mathrm{~Hz}\right), 119.6,30.0$ $\left(\mathrm{d}, J_{\mathrm{P}-\mathrm{C}}=14.5 \mathrm{~Hz}\right), 29.5\left(\mathrm{~d}, J_{\mathrm{P}-\mathrm{C}}=71.2 \mathrm{~Hz}\right), 28.1,25.0,21.2\left(\mathrm{~d}, J_{\mathrm{P}-\mathrm{C}}=4.1 \mathrm{~Hz}\right), 17.0 ;{ }^{31} \mathrm{P} \mathrm{NMR}$ (201.95 MHz, $\mathrm{CDCl}_{3}$ ) $\delta 32.3$; mass spectrum (EI) m/e $311(\mathrm{M}+, 29), 312(100)$; HRMS $\left(\mathrm{M}^{+}\right)$ calcd for $\mathrm{C}_{19} \mathrm{H}_{22} \mathrm{~N}_{1} \mathrm{O}_{1} \mathrm{P}_{1}$ 311.1439, found 311.1437. Anal. Calcd for $\mathrm{C}_{19} \mathrm{H}_{22} \mathrm{~N}_{1} \mathrm{O}_{1} \mathrm{P}_{1}$ : C, 73.29; $\mathrm{H}$, 
7.12; N, 4.50. Found: C, 73.61; H, 7.38; N, 4.10.

$\mathrm{Ph}_{2}(\mathrm{O}) \mathrm{P} \leadsto\left(\mathrm{CH}_{2}\right)_{4} \mathrm{CO}_{2} \mathrm{Et}$

(Table 2, run 6)

Colorless oil; ${ }^{1} \mathrm{H}$ NMR (499.1 MHz, $\left.\mathrm{CDCl}_{3}\right) \delta$ 7.76-7.72 (m, 4H), 7.52-7.44 (m, 6H), 2.29-2.23 (m, 2H), 1.67-1.54 (m, 4H), 1.67-1.54 (m, 4H), 1.42 (quint, $J=7.5 \mathrm{~Hz}, 2 \mathrm{H}), 1.33-1.21(\mathrm{~m}, 6 \mathrm{H})$; ${ }^{13} \mathrm{C}$ NMR (125.4 MHz, $\left.\mathrm{CDCl}_{3}\right) \delta 173.4,133.0\left(\mathrm{~d}, J_{\mathrm{P}-\mathrm{C}}=98.2 \mathrm{~Hz}\right), 131.5\left(\mathrm{~d}, J_{\mathrm{P}-\mathrm{C}}=2.0 \mathrm{~Hz}\right), 130.6$ $\left(\mathrm{d}, J_{\mathrm{P}-\mathrm{C}}=9.3 \mathrm{~Hz}\right), 128.5\left(\mathrm{~d}, J_{\mathrm{P}-\mathrm{C}}=11.4 \mathrm{~Hz}\right), 60.0,34.0,30.4\left(\mathrm{~d}, J_{\mathrm{P}-\mathrm{C}}=14.4 \mathrm{~Hz}\right), 29.5\left(\mathrm{~d}, J_{\mathrm{P}-\mathrm{C}}=\right.$ 71.4 Hz), 28.4, 24.5, 21.1 (d, $J_{\mathrm{P}-\mathrm{C}}=3.0 \mathrm{~Hz}$ ), 14.1; ${ }^{31} \mathrm{P} \mathrm{NMR}\left(201.95 \mathrm{MHz}, \mathrm{CDCl}_{3}\right.$ ) $\delta 32.4$; mass spectrum (EI) m/e $358\left(\mathrm{M}+\right.$, 35), 381 (100); HRMS ( $\mathrm{M}^{+}$) calcd for $\mathrm{C}_{21} \mathrm{H}_{27} \mathrm{O}_{3} \mathrm{P}_{1}$ 358.1698, found 358.1687. Anal. Calcd for $\mathrm{C}_{21} \mathrm{H}_{27} \mathrm{O}_{3} \mathrm{P}_{1}$ : C, 70.37; H, 7.59. Found: C, 69.97; H, 7.40.

(Table 2, run 7)<smiles>CCCCCP(=O)(c1ccccc1)c1ccccc1</smiles>

(Table 2, run 7)

White solid, mp: $110-110.5{ }^{\circ} \mathrm{C} ;{ }^{1} \mathrm{H}$ NMR (499.1 MHz, $\left.\mathrm{CDCl}_{3}\right) \delta$ 7.22-7.18 (m, 4H), 6.99-6.91 (m, $6 \mathrm{H}), 1.66-1.60$ (m, 2H), 0.36 (dd, $\left.J_{\mathrm{H}-\mathrm{H}}=7.9 \mathrm{~Hz}, J_{\mathrm{P}-\mathrm{H}}=0.9 \mathrm{~Hz}, 6 \mathrm{H}\right), 0.26-0.21(\mathrm{~m}, 2 \mathrm{H}), 0.009$ (dd, $\left.J_{\mathrm{H}-\mathrm{H}}=7.9 \mathrm{~Hz}, J_{\mathrm{P}-\mathrm{H}}=1.2 \mathrm{~Hz}, 9 \mathrm{H}\right) ;{ }^{13} \mathrm{C} \mathrm{NMR}\left(125.4 \mathrm{MHz}, \mathrm{CDCl}_{3}\right) \delta 133.0\left(\mathrm{~d}, J_{\mathrm{P}-\mathrm{C}}=96.2 \mathrm{~Hz}\right)$, $131.6\left(\mathrm{~d}, J_{\mathrm{P}-\mathrm{C}}=2.1 \mathrm{~Hz}\right), 130.9\left(\mathrm{~d}, J_{\mathrm{P}-\mathrm{C}}=9.3 \mathrm{~Hz}\right), 128.6\left(\mathrm{~d}, J_{\mathrm{P}-\mathrm{C}}=11.3 \mathrm{~Hz}\right), 23.8\left(\mathrm{~d}, J_{\mathrm{P}-\mathrm{C}}=69.2 \mathrm{~Hz}\right)$, 7.3, 2.9, 1.7 (d, $\left.J_{\mathrm{P}-\mathrm{C}}=6.3 \mathrm{~Hz}\right) ;{ }^{31} \mathrm{P}$ NMR $\left(201.95 \mathrm{MHz}, \mathrm{CDCl}_{3}\right) \delta 34.3$; mass spectrum (EI) m/e $344(\mathrm{M}+, 90), 331$ (100); HRMS $\left(\mathrm{M}^{+}\right)$calcd for $\mathrm{C}_{20} \mathrm{H}_{29} \mathrm{O}_{1} \mathrm{P}_{1} \mathrm{Si}_{1}$ 344.1731, found 344.1725. Anal. Calcd for $\mathrm{C}_{20} \mathrm{H}_{29} \mathrm{O}_{1} \mathrm{P}_{1} \mathrm{Si}_{1}$ : C, 69.73; H, 8.48. Found: C, 69.56; H, 8.31.

$\mathrm{Ph}_{2}(\mathrm{O}) \mathrm{P} ح \mathrm{O}-\mathrm{Bu}$

(Table 2, run 10)

Colorless oil; ${ }^{1} \mathrm{H}$ NMR (499.1 MHz, $\left.\mathrm{CDCl}_{3}\right) \delta$ 7.77-7.73 (m, 4H), 7.53-7.44 (m, 6H), 3.77-3.72 (m, 2H), 3.35-3.32 (m, 2H), 2.68-2.62 (m, 2H), 1.45-1.39 (m, 2H), 1.28-1.20 (m, 2H), 0.86-0.83 (m, 3H); ${ }^{13} \mathrm{C}$ NMR (125.4 MHz, $\left.\mathrm{CDCl}_{3}\right) \delta 133.0\left(\mathrm{~d}, J_{\mathrm{P}-\mathrm{C}}=99.2 \mathrm{~Hz}\right), 131.6\left(\mathrm{~d}, J_{\mathrm{P}-\mathrm{C}}=2.0 \mathrm{~Hz}\right)$, $130.6\left(\mathrm{~d}, J_{\mathrm{P}-\mathrm{C}}=9.3 \mathrm{~Hz}\right), 128.5\left(\mathrm{~d}, J_{\mathrm{P}-\mathrm{C}}=12.4 \mathrm{~Hz}\right), 70.7,63.9,31.5,30.4\left(\mathrm{~d}, J_{\mathrm{P}-\mathrm{C}}=70.2 \mathrm{~Hz}\right), 19.1$, 13.7; ${ }^{31} \mathrm{P}$ NMR (201.95 MHz, $\mathrm{CDCl}_{3}$ ) $\delta$ 29.7; mass spectrum (EI) m/e 302 (M+, 27), 281 (100); HRMS $\left(\mathrm{M}^{+}\right.$) calcd for $\mathrm{C}_{18} \mathrm{H}_{23} \mathrm{O}_{2} \mathrm{P}$ 302.1436, found 302.1432. Anal. Calcd for $\mathrm{C}_{18} \mathrm{H}_{23} \mathrm{O}_{2} \mathrm{P}$ : C, 71.50; H, 7.67. Found: C, 71.23; H, 7.61.<smiles>O=S(CCS(=O)c1ccccc1)c1ccccc1</smiles>

(Table 2, run 11)

White solid, mp: $110.5-113{ }^{\circ} \mathrm{C} ;{ }^{1} \mathrm{H}$ NMR (499.1 MHz, $\mathrm{CDCl}_{3}$ ) $\delta$ 7.74-7.42 (m, 15H), 3.26-3.20 (m, 
1H), 2.89-2.75 (m, 2H), 2.35-2.28 (m, 1H); $\left.{ }^{13} \mathrm{C} \mathrm{NMR} \mathrm{(125.4} \mathrm{MHz,} \mathrm{CDCl}_{3}\right) \delta 142.3,132.25(\mathrm{~d}$, $\left.J_{\mathrm{P}-\mathrm{C}}=16.6 \mathrm{~Hz}\right), 132.24\left(\mathrm{~d}, J_{\mathrm{P}-\mathrm{C}}=2.0 \mathrm{~Hz}\right), 132.1\left(\mathrm{~d}, J_{\mathrm{P}-\mathrm{C}}=3.0 \mathrm{~Hz}\right), 131.2,130.8\left(\mathrm{~d}, J_{\mathrm{P}-\mathrm{C}}=9.3 \mathrm{~Hz}\right)$, $130.7\left(\mathrm{~d}, J_{\mathrm{P}-\mathrm{C}}=9.3 \mathrm{~Hz}\right), 129.0\left(\mathrm{~d}, J_{\mathrm{P}-\mathrm{C}}=10.3 \mathrm{~Hz}\right), 128.9\left(\mathrm{~d}, J_{\mathrm{P}-\mathrm{C}}=11.4 \mathrm{~Hz}\right), 124.0,48.0,21.1$ (d, $\left.J_{\mathrm{P}-\mathrm{C}}=69.2 \mathrm{~Hz}\right) ;{ }^{31} \mathrm{P}$ NMR $\left(201.95 \mathrm{MHz}, \mathrm{CDCl}_{3}\right) \delta 31.4$; mass spectrum (EI) m/e $354(\mathrm{M}+, 28)$, 331 (100); HRMS $\left(\mathrm{M}^{+}\right)$calcd for $\mathrm{C}_{20} \mathrm{H}_{19} \mathrm{O}_{2} \mathrm{P}_{1} \mathrm{~S}_{1}$ 354.0843, found 354.0842. Anal. Calcd for $\mathrm{C}_{20} \mathrm{H}_{19} \mathrm{O}_{2} \mathrm{P}_{1} \mathrm{~S}_{1}$ : 67.78; H, 5.40. Found: C, 67.91; H, 5.31.

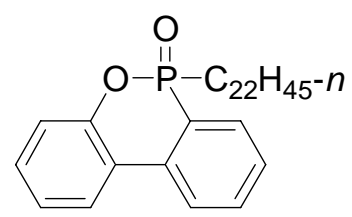

(Table 2, run 17)

White solid, mp: 69.5-70 ${ }^{\circ} \mathrm{C} ;{ }^{1} \mathrm{H}$ NMR (499.1 MHz, $\left.\mathrm{CDCl}_{3}\right) \delta$ 7.96-7.84 (m, 3H), 7.69-7.66 (m, 1H), 7.52-7.48 (m, 1H), 7.38-7.34 (m, 1H), 7.25-7.21 (m, 1H), 2.10-1.97 (m, 2H), 1.66-1.56 (m, 2H), $1.35-1.21(\mathrm{~m}, 38 \mathrm{H}) 0.88(\mathrm{t}, J=7.0 \mathrm{~Hz}, 3 \mathrm{H}) ;{ }^{13} \mathrm{C} \mathrm{NMR}\left(125.4 \mathrm{MHz}, \mathrm{CDCl}_{3}\right) \delta 149.3\left(\mathrm{~d}, J_{\mathrm{P}-\mathrm{C}}=\right.$ $8.3 \mathrm{~Hz}$ ), 135.6 (d, $\left.J_{\mathrm{P}-\mathrm{C}}=6.1 \mathrm{~Hz}\right), 133.1$ (d, $\left.J_{\mathrm{P}-\mathrm{C}}=2.0 \mathrm{~Hz}\right), 130.5,130.0\left(\mathrm{~d}, J_{\mathrm{P}-\mathrm{C}}=10.3 \mathrm{~Hz}\right), 128.4$ $\left(\mathrm{d}, J_{\mathrm{P}-\mathrm{C}}=12.4 \mathrm{~Hz}\right), 125.1,124.9\left(\mathrm{~d}, J_{\mathrm{P}-\mathrm{C}}=115.7 \mathrm{~Hz}\right), 124.5,123.8\left(\mathrm{~d}, J_{\mathrm{P}-\mathrm{C}}=9.3 \mathrm{~Hz}\right), 122.3\left(\mathrm{~d}, J_{\mathrm{P}-\mathrm{C}}\right.$ $=11.4 \mathrm{~Hz}), 120.4\left(\mathrm{~d}, J_{\mathrm{P}-\mathrm{C}}=6.1 \mathrm{~Hz}\right), 31.9,30.6$ (d, $\left.J_{\mathrm{P}-\mathrm{C}}=15.4 \mathrm{~Hz}\right), 29.7$ (sixteen peaks overlapped), $29.6\left(\mathrm{~d}, J_{\mathrm{P}-\mathrm{C}}=9.4 \mathrm{~Hz}\right), 29.3\left(\mathrm{~d}, J_{\mathrm{P}-\mathrm{C}}=9.43 \mathrm{~Hz}\right), 29.0,28.6\left(\mathrm{~d}, J_{\mathrm{P}-\mathrm{C}}=96.1 \mathrm{~Hz}\right) ;{ }^{31} \mathrm{P}$ NMR $(201.95$ $\left.\mathrm{MHz}, \mathrm{CDCl}_{3}\right) \delta$ 38.6; mass spectrum (EI) m/e $524(\mathrm{M}+, 100)$; HRMS $\left(\mathrm{M}^{+}\right)$calcd for $\mathrm{C}_{34} \mathrm{H}_{53} \mathrm{O}_{2} \mathrm{P}_{1}$ 524.3783, found 524.3790. Anal. Calcd for $\mathrm{C}_{20} \mathrm{H}_{19} \mathrm{O}_{2} \mathrm{P}_{1} \mathrm{~S}_{1}$ : C, 77.82; $\mathrm{H}, 10.18$. Found: $\mathrm{C}, 77.67 ; \mathrm{H}$, 10.31 .



(Table 2, run 18)

White solid, mp: $210-211{ }^{\circ} \mathrm{C} ;{ }^{1} \mathrm{H}$ NMR (499.1 MHz, $\left.\mathrm{CDCl}_{3}\right) \delta$ 7.93-7.17 (m, 18H), 2.66-2.53 (m, 2H), 2.33-2.17 (m, 2H); ${ }^{13} \mathrm{C}$ NMR (125.4 MHz, $\left.\mathrm{CDCl}_{3}\right) \delta 148.7$ (d, $\left.J_{\mathrm{P}-\mathrm{C}}=7.1 \mathrm{~Hz}\right), 135.7$ (d, $J_{\mathrm{P}-\mathrm{C}}$ $=6.3 \mathrm{~Hz}), 133.5\left(\mathrm{~d}, J_{\mathrm{P}-\mathrm{C}}=2.0 \mathrm{~Hz}\right), 132.0\left(\mathrm{~d}, J_{\mathrm{P}-\mathrm{C}}=3.1 \mathrm{~Hz}\right), 131.5\left(\mathrm{~d}, J_{\mathrm{P}-\mathrm{C}}=73.4 \mathrm{~Hz}\right), 130.8(\mathrm{~d}$, $\left.J_{\mathrm{P}-\mathrm{C}}=47.5 \mathrm{~Hz}\right), 130.6\left(\mathrm{~d}, J_{\mathrm{P}-\mathrm{C}}=3.1 \mathrm{~Hz}\right), 130.5\left(\mathrm{~d}, J_{\mathrm{P}-\mathrm{C}}=3.0 \mathrm{~Hz}\right), 129.9\left(\mathrm{~d}, J_{\mathrm{P}-\mathrm{C}}=10.4 \mathrm{~Hz}\right), 128.7$ $\left(\mathrm{d}, J_{\mathrm{P}-\mathrm{C}}=11.4 \mathrm{~Hz}\right), 128.4\left(\mathrm{~d}, J_{\mathrm{P}-\mathrm{C}}=13.4 \mathrm{~Hz}\right), 124.9\left(\mathrm{~d}, J_{\mathrm{P}-\mathrm{C}}=55.8 \mathrm{~Hz}\right), 123.9\left(\mathrm{~d}, J_{\mathrm{P}-\mathrm{C}}=9.4 \mathrm{~Hz}\right)$, $123.5\left(\mathrm{~d}, J_{\mathrm{P}-\mathrm{C}}=118.9 \mathrm{~Hz}\right), 122.0\left(\mathrm{~d}, J_{\mathrm{P}-\mathrm{C}}=10.3 \mathrm{~Hz}\right), 120.1\left(\mathrm{~d}, J_{\mathrm{P}-\mathrm{C}}=5.1 \mathrm{~Hz}\right), 21.7\left(\mathrm{dd}, J_{\mathrm{P}-\mathrm{C}}=69.7\right.$ $\left.\mathrm{Hz}, J_{\mathrm{P}-\mathrm{C}}=4.1 \mathrm{~Hz}\right), 20.6\left(\mathrm{~d}, J_{\mathrm{P}-\mathrm{C}}=96.0 \mathrm{~Hz}, \quad J_{\mathrm{P}-\mathrm{C}}=4.1 \mathrm{~Hz}\right), 16.2\left(\mathrm{~d}, J_{\mathrm{P}-\mathrm{C}}=5.1 \mathrm{~Hz}\right) ;{ }^{31} \mathrm{P} \mathrm{NMR}$ $\left(201.95 \mathrm{MHz}, \mathrm{CDCl}_{3}\right) \delta 37.0$ (d, $J_{\mathrm{P}-\mathrm{P}}=58.2 \mathrm{~Hz}$ ), 31.9 (d, $\left.J_{\mathrm{P}-\mathrm{P}}=59.6 \mathrm{~Hz}\right)$; mass spectrum (EI) m/e $444(\mathrm{M}+, 83), 431$ (100); exact mass $\left(\mathrm{M}^{+}\right)$calcd for $\mathrm{C}_{26} \mathrm{H}_{22} \mathrm{O}_{3} \mathrm{P}_{2}$ 444.1044, found 444.1049. Anal. Calcd for $\mathrm{C}_{26} \mathrm{H}_{22} \mathrm{O}_{3} \mathrm{P}_{2}$ : C, 70.27; $\mathrm{H}, 4.99$. Found: C, 70.12; H, 4.56. 


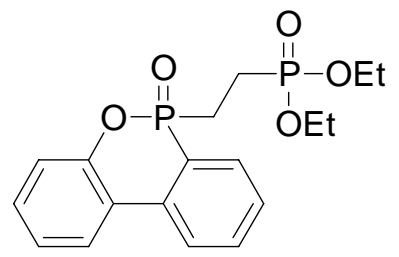

(Table 2, run 19)

Colorless oil; ${ }^{1} \mathrm{H}$ NMR (499.1 MHz, $\left.\mathrm{CDCl}_{3}\right) \delta$ 7.99-7.96 (m, 1H), 7.93-7.91 (m, 1H), 7.89-7.85 (m, 1H), 7.73-7.70 (m, 1H), 7.73-7.70 (m, 1H), 7.54-7.51 (m, 1H), 7.40-7.37 (m, 1H), 7.28-7.22 (m, 1H), 4.08-4,04 (m, 4H), 2.35-2.21 (m, 2H), 2.09-1.90 (m, 2H), 1.27 (dd, $J_{\mathrm{H}-\mathrm{H}}=7.0 \mathrm{~Hz}, J_{\mathrm{P}-\mathrm{H}}$ $=1.2 \mathrm{~Hz}, 6 \mathrm{H}) ;{ }^{13} \mathrm{C}$ NMR $\left(125.4 \mathrm{MHz}, \mathrm{CDCl}_{3}\right) \delta 148.9\left(\mathrm{~d}, J_{\mathrm{P}-\mathrm{C}}=8.3 \mathrm{~Hz}\right), 135.7\left(\mathrm{~d}, J_{\mathrm{P}-\mathrm{C}}=6.1 \mathrm{~Hz}\right)$, $133.5\left(\mathrm{~d}, J_{\mathrm{P}-\mathrm{C}}=2.1 \mathrm{~Hz}\right), 130.6,129.9\left(\mathrm{~d}, J_{\mathrm{P}-\mathrm{C}}=10.4 \mathrm{~Hz}\right), 128.5\left(\mathrm{~d}, J_{\mathrm{P}-\mathrm{C}}=13.4 \mathrm{~Hz}\right), 124.9\left(\mathrm{~d}, J_{\mathrm{P}-\mathrm{C}}=\right.$ $51.7 \mathrm{~Hz}), 123.9\left(\mathrm{~d}, J_{\mathrm{P}-\mathrm{C}}=9.4 \mathrm{~Hz}\right), 123.4\left(\mathrm{~d}, J_{\mathrm{P}-\mathrm{C}}=120.9 \mathrm{~Hz}\right), 121.9\left(\mathrm{~d}, J_{\mathrm{P}-\mathrm{C}}=10.4 \mathrm{~Hz}\right), 120.2(\mathrm{~d}$, $\left.J_{\mathrm{P}-\mathrm{C}}=6.1 \mathrm{~Hz}\right), 61.91\left(\mathrm{~d}, J_{\mathrm{P}-\mathrm{C}}=2.0 \mathrm{~Hz}\right), 61.86\left(\mathrm{~d}, J_{\mathrm{P}-\mathrm{C}}=3.1 \mathrm{~Hz}\right), 21.9\left(\mathrm{dd}, J_{\mathrm{P}-\mathrm{C}}=97.2 \mathrm{~Hz}, J_{\mathrm{P}-\mathrm{C}}=5.1\right.$ $\mathrm{Hz}), 18.0\left(\mathrm{~d}, J_{\mathrm{P}-\mathrm{C}}=144.6 \mathrm{~Hz}, \quad J_{\mathrm{P}-\mathrm{C}}=5.2 \mathrm{~Hz}\right), 16.2\left(\mathrm{~d}, J_{\mathrm{P}-\mathrm{C}}=5.1 \mathrm{~Hz}\right) ;{ }^{31} \mathrm{P}$ NMR $(201.95 \mathrm{MHz}$, $\left.\mathrm{CDCl}_{3}\right) \delta 36.2\left(\mathrm{~d}, J_{\mathrm{P}-\mathrm{P}}=75.5 \mathrm{~Hz}\right), 29.1\left(\mathrm{~d}, J_{\mathrm{P}-\mathrm{P}}=75.3 \mathrm{~Hz}\right.$ ); mass spectrum (EI) m/e $380(\mathrm{M}+$, 100); HRMS $\left(\mathrm{M}^{+}\right)$calcd for $\mathrm{C}_{18} \mathrm{H}_{22} \mathrm{O}_{5} \mathrm{P}_{2}$ 380.0942, found 380.0943. Anal. Calcd for $\mathrm{C}_{18} \mathrm{H}_{22} \mathrm{O}_{5} \mathrm{P}_{2}$ : C, 56.85; H, 5.83. Found: C, 56.13; H, 5.23.

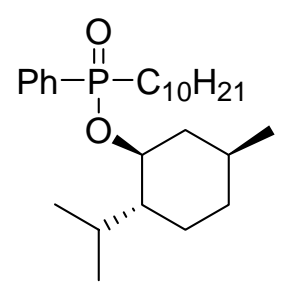

(equation 1)

Colorless oil; ${ }^{1} \mathrm{H}$ NMR (499.1 MHz, $\left.\mathrm{CDCl}_{3}\right) \delta$ 7.80-7.77 (m, 2H), 7.52-7.44 (m, 3H), 4.31-4.25 (m, 1H), 2.27-2.24 (m, 2H), 1.89-1.27 (m, 23H), 1.02-0.72 (m, 16H); ${ }^{13} \mathrm{C}$ NMR (125.4 MHz, $\left.\mathrm{CDCl}_{3}\right) \delta 133.3\left(\mathrm{~d}, J_{\mathrm{P}-\mathrm{C}}=123.0 \mathrm{~Hz}\right), 131.5\left(\mathrm{~d}, J_{\mathrm{P}-\mathrm{C}}=2.0 \mathrm{~Hz}\right), 131.3\left(\mathrm{~d}, J_{\mathrm{P}-\mathrm{C}}=10.3 \mathrm{~Hz}\right), 128.3(\mathrm{~d}$, $\left.J_{\mathrm{P}-\mathrm{C}}=12.4 \mathrm{~Hz}\right), 48.9\left(\mathrm{~d}, J_{\mathrm{P}-\mathrm{C}}=5.1 \mathrm{~Hz}\right), 43.2,34.1,31.9,31.4,30.9,30.8,30.7,30.1,29.5,29.33$, 29.27, 29.1, 25.7, 22.8 (d, $J_{\mathrm{P}-\mathrm{C}}=30.0 \mathrm{~Hz}$ ), 21.7 (d, $J_{\mathrm{P}-\mathrm{C}}=3.0 \mathrm{~Hz}$ ), 21.5 (d, $\left.J_{\mathrm{P}-\mathrm{C}}=99.0 \mathrm{~Hz}\right), 15.8$, 14.1; ${ }^{31} \mathrm{P}$ NMR (201.95 MHz, $\mathrm{CDCl}_{3}$ ) $\delta 42.4$; mass spectrum (EI) m/e 420 (M+, 56), 405 (100); HRMS $\left(\mathrm{M}^{+}\right)$calcd for $\mathrm{C}_{26} \mathrm{H}_{45} \mathrm{O}_{2} \mathrm{P}_{1}$ 420.3157, found 420.3157. Anal. Calcd for $\mathrm{C}_{26} \mathrm{H}_{45} \mathrm{O}_{2} \mathrm{P}_{1}$ : C, 74.24; H, 10.78. Found: C, 74.11; H, 10.45. 


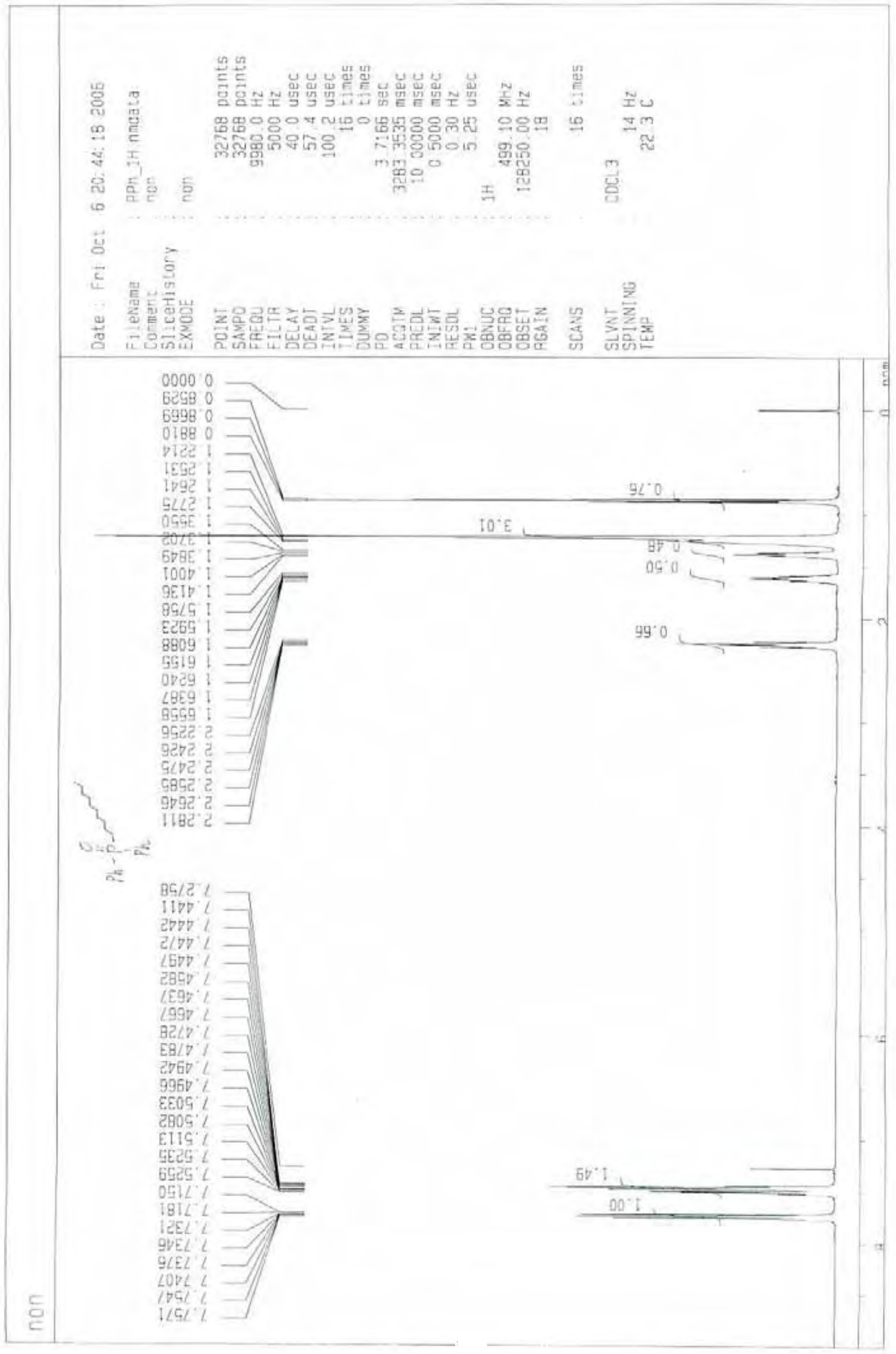




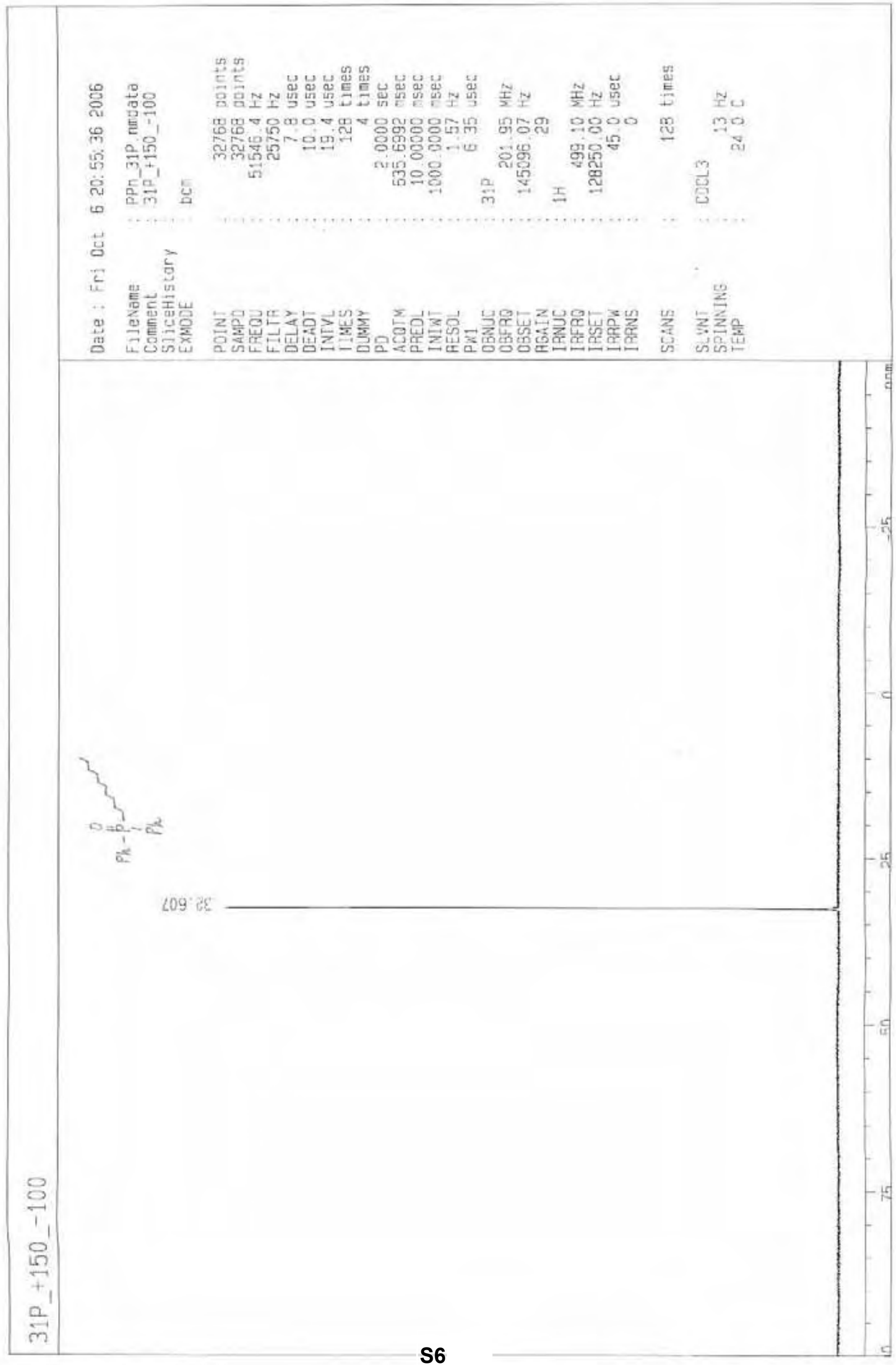




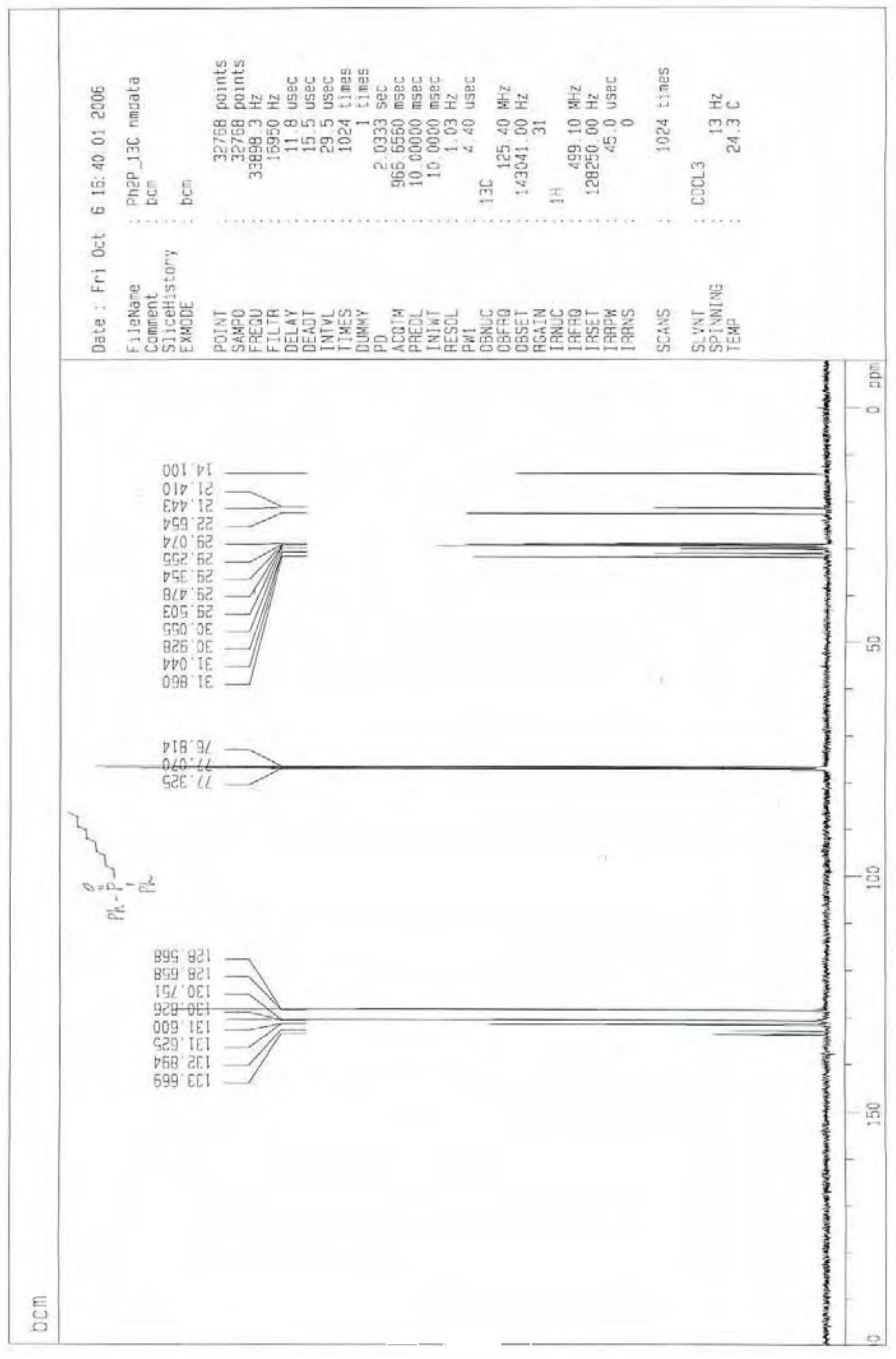




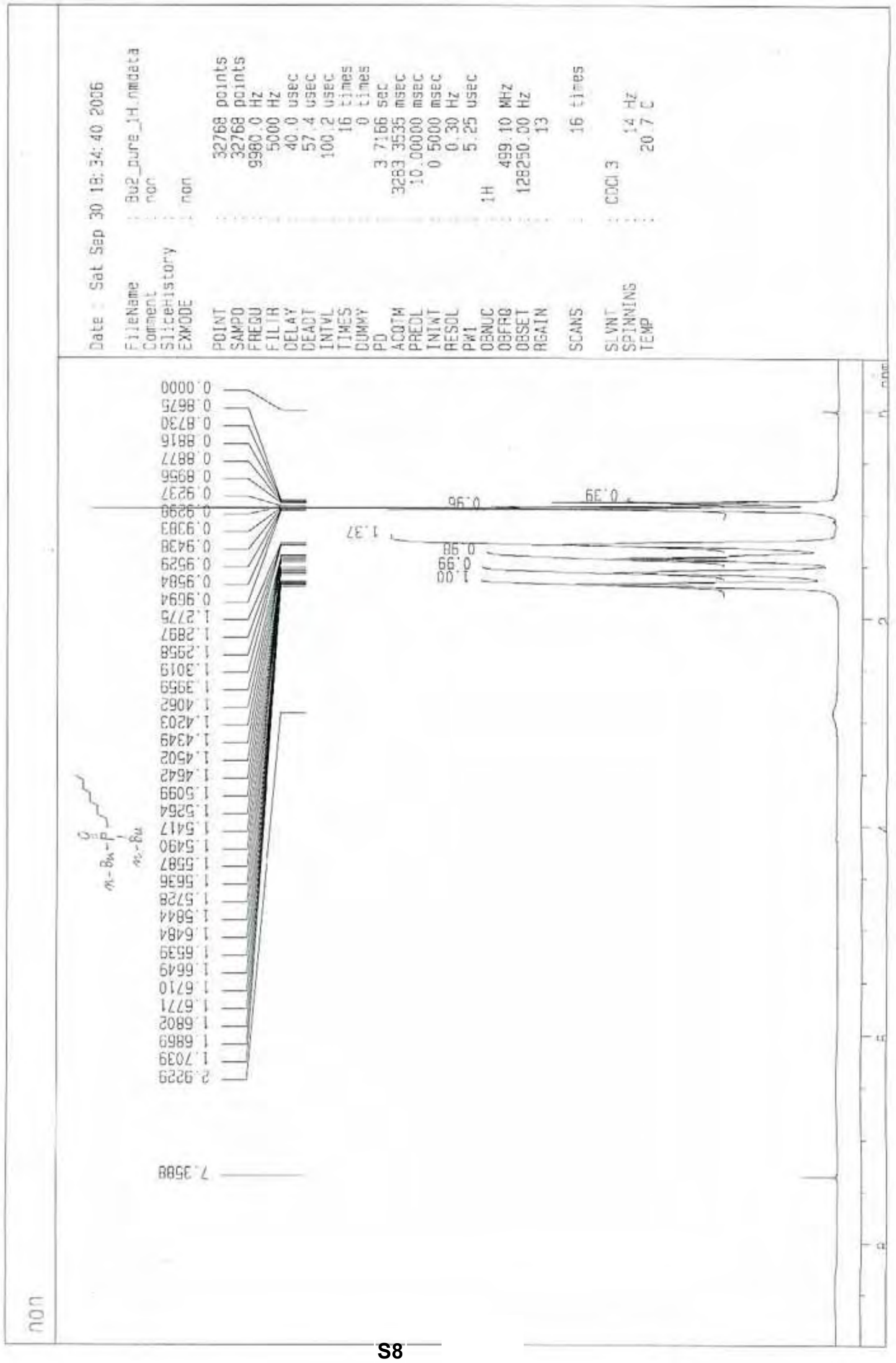




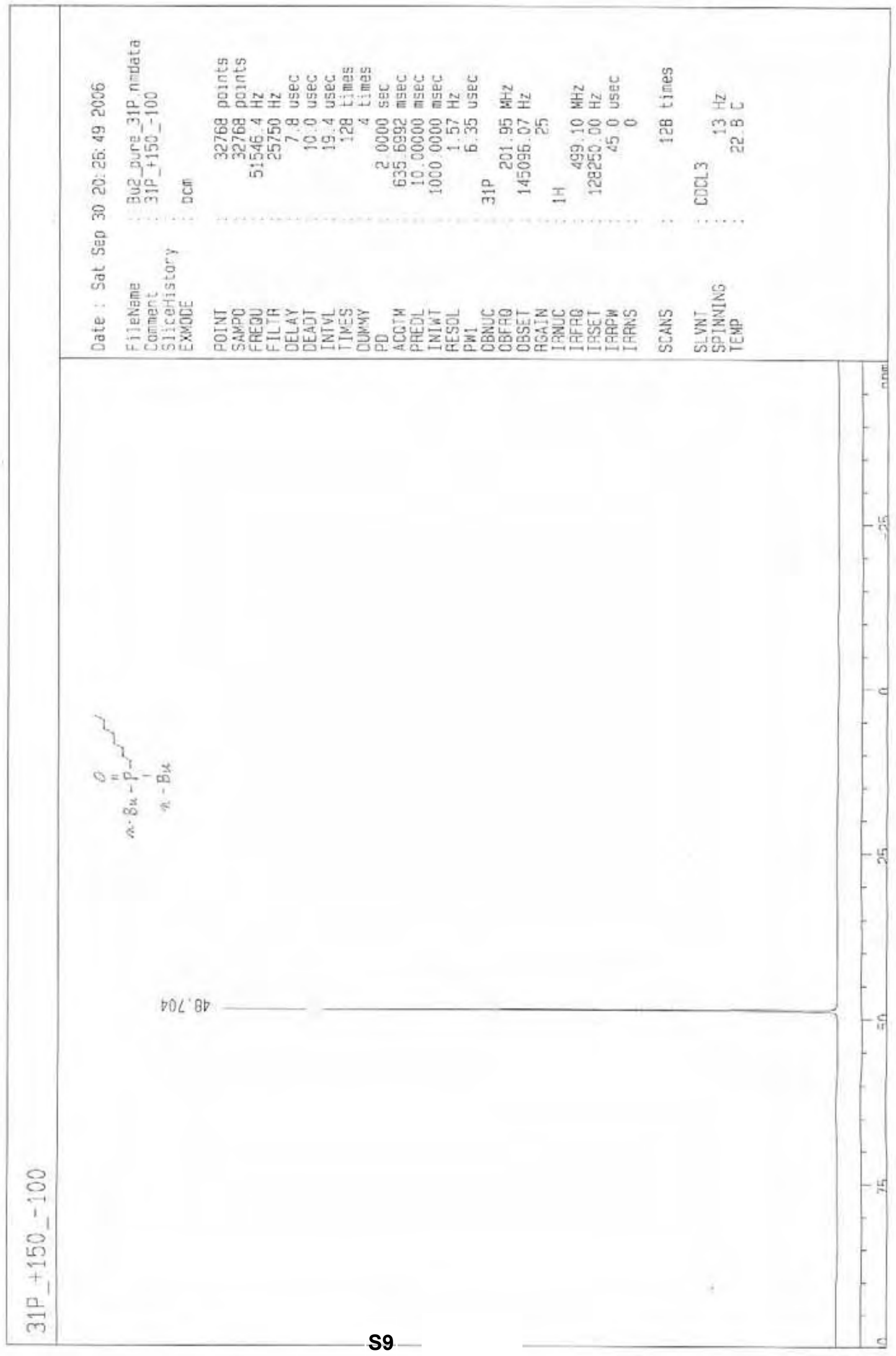




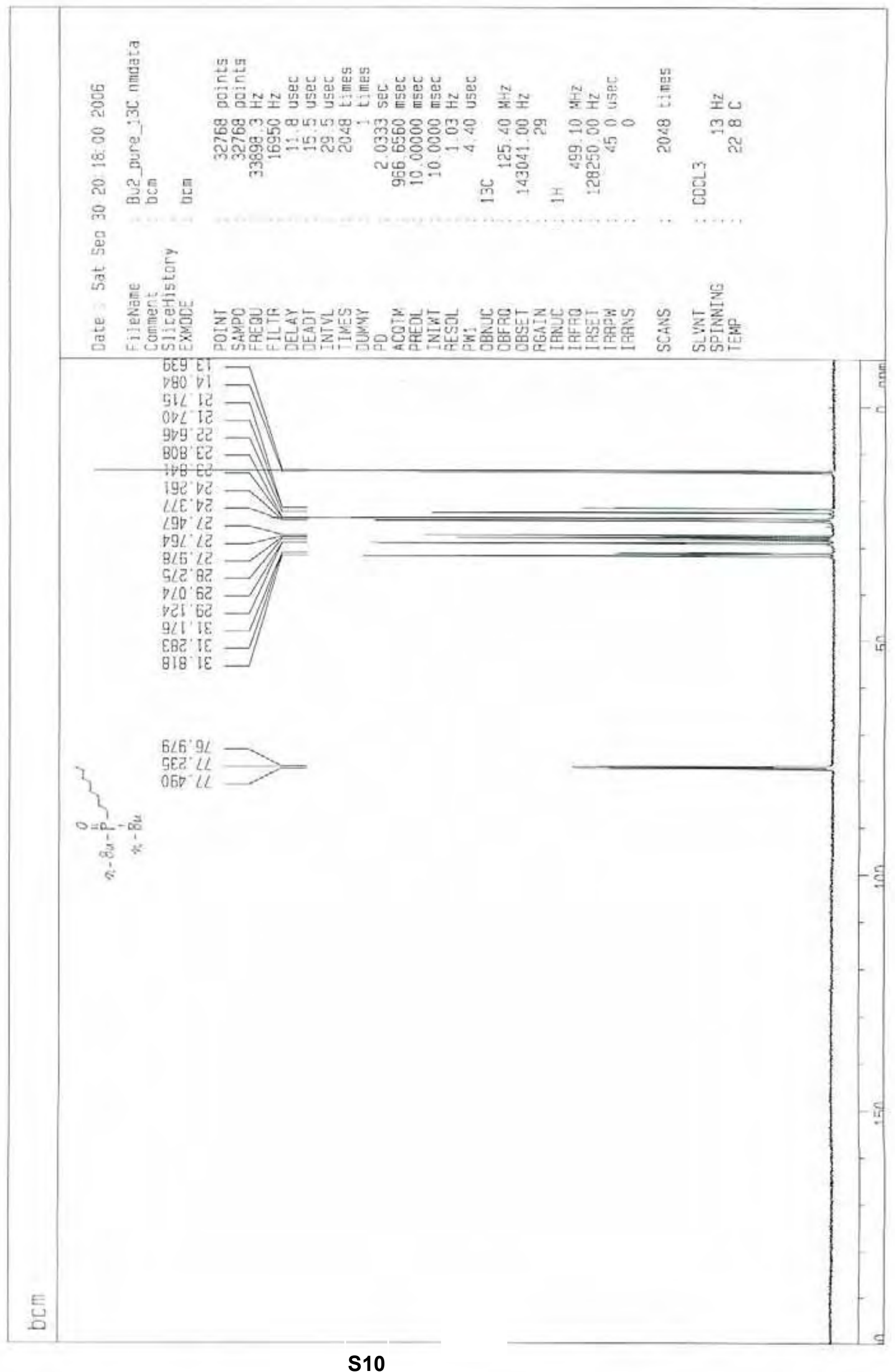




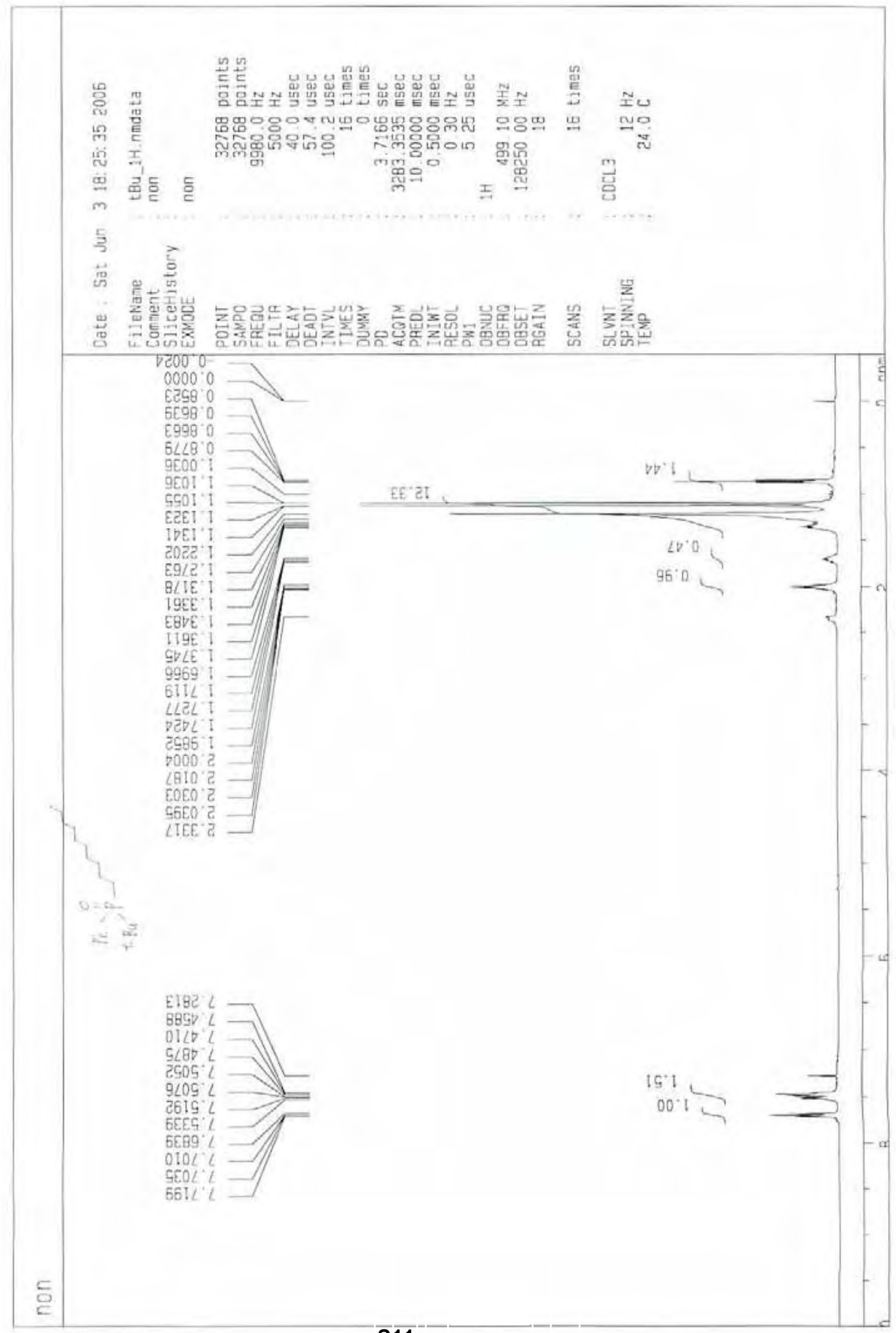




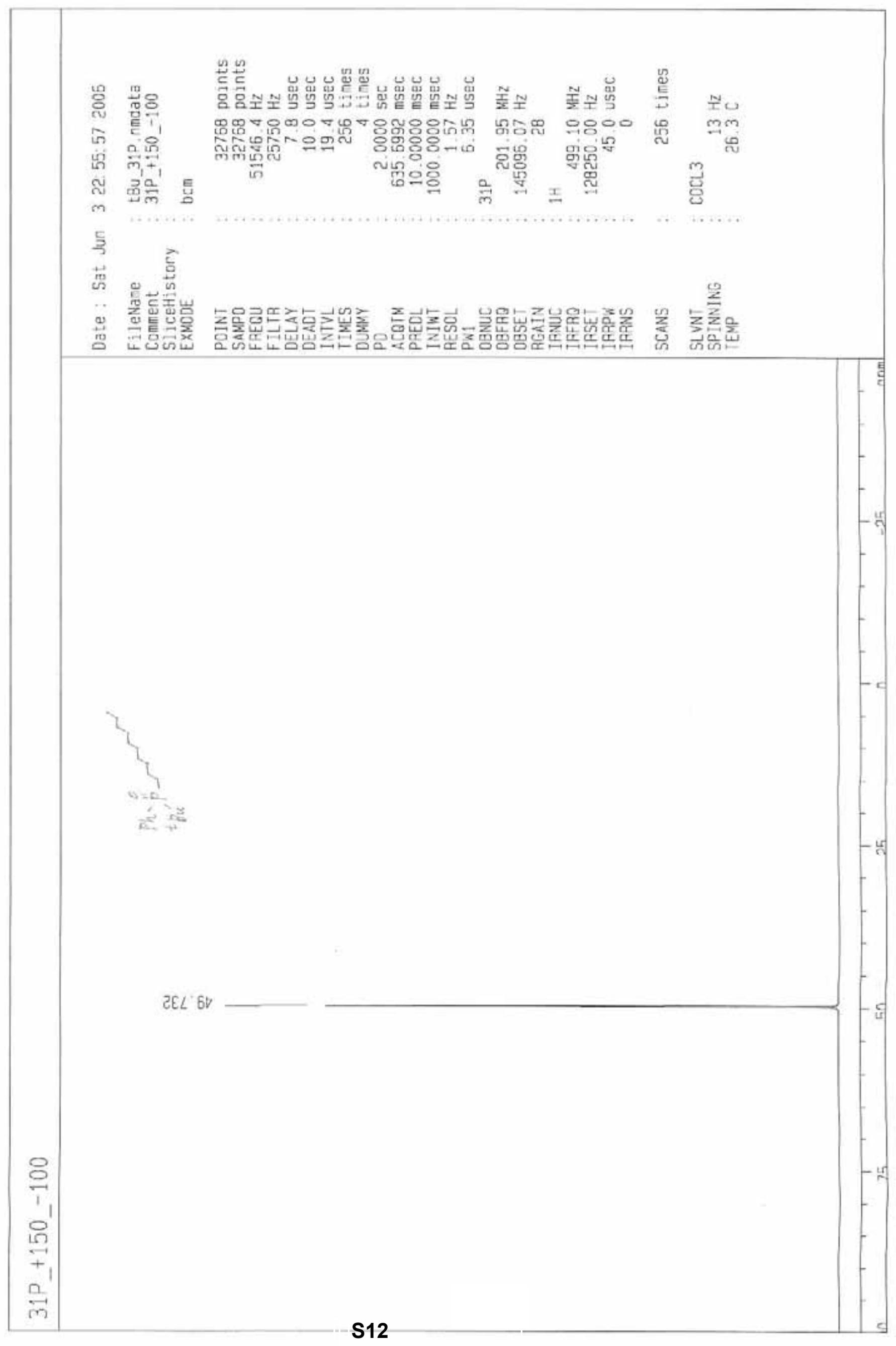




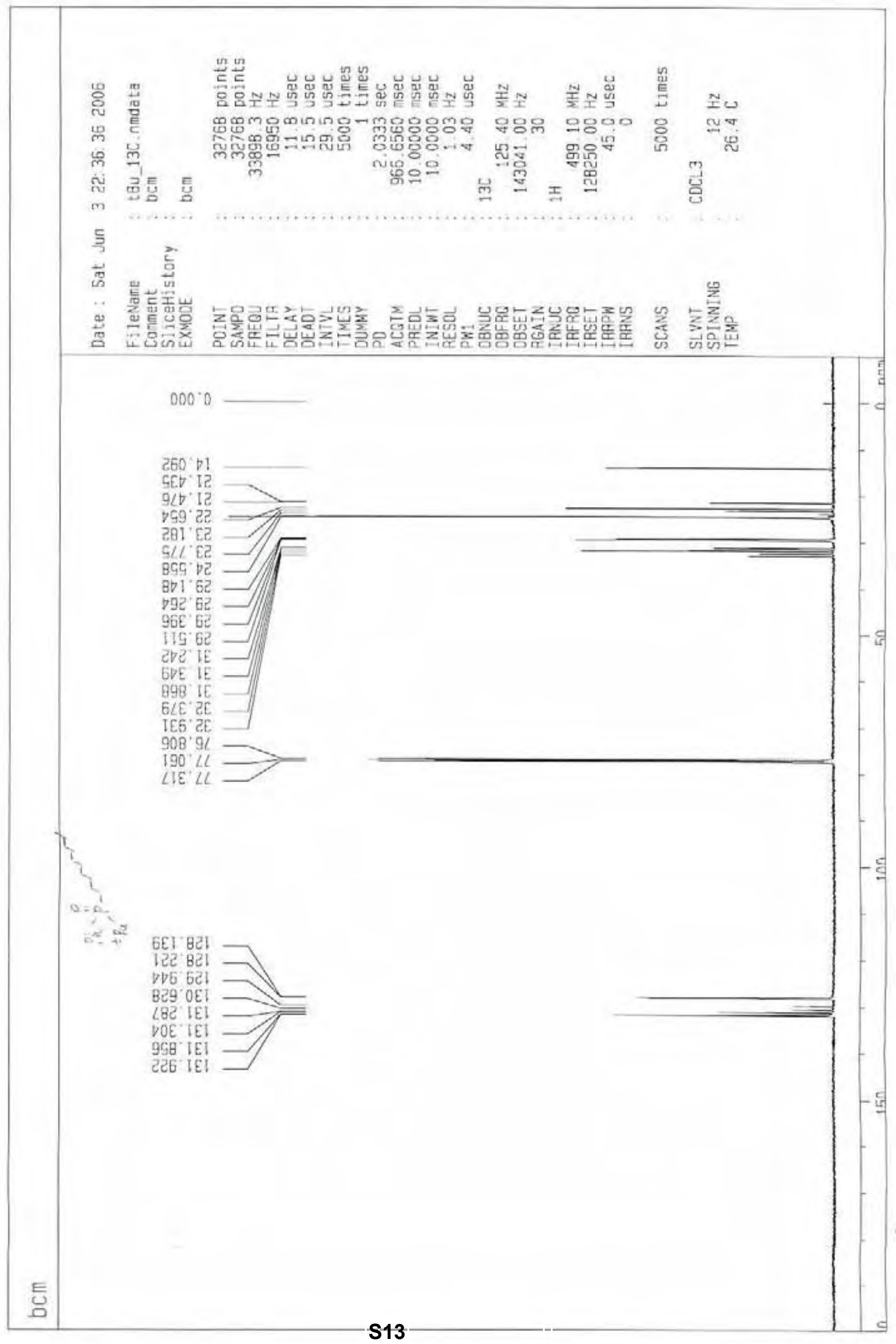









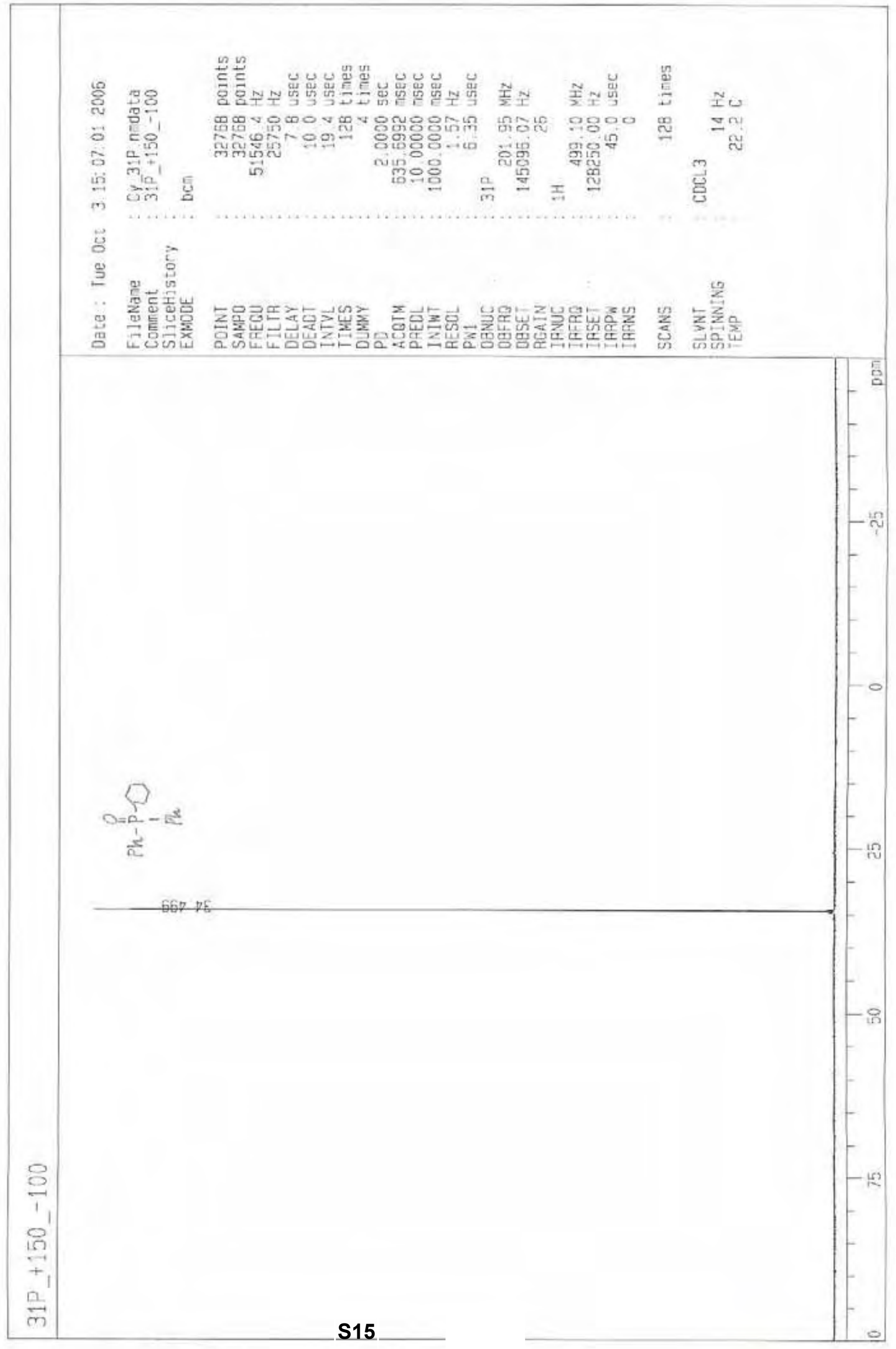




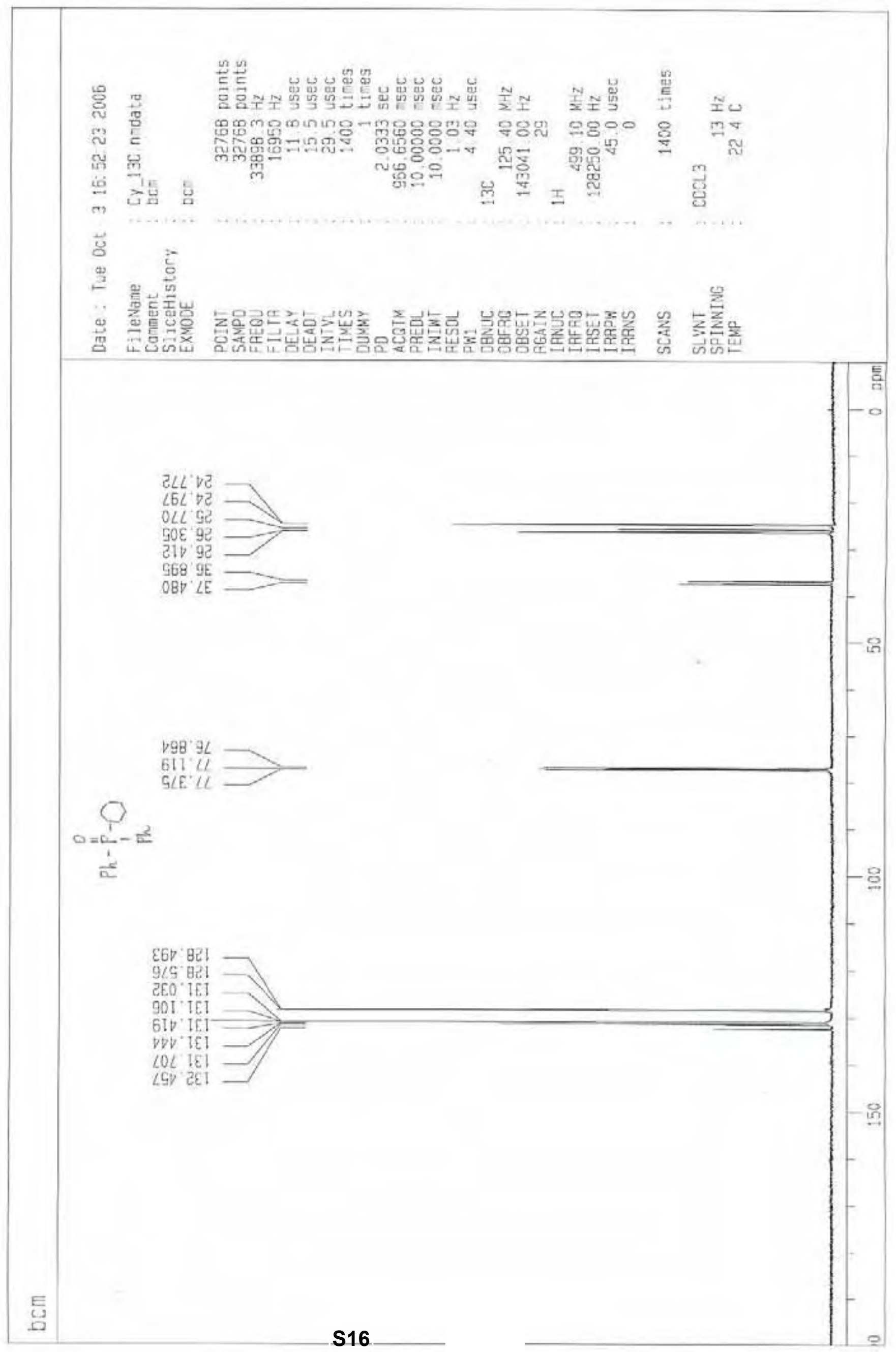




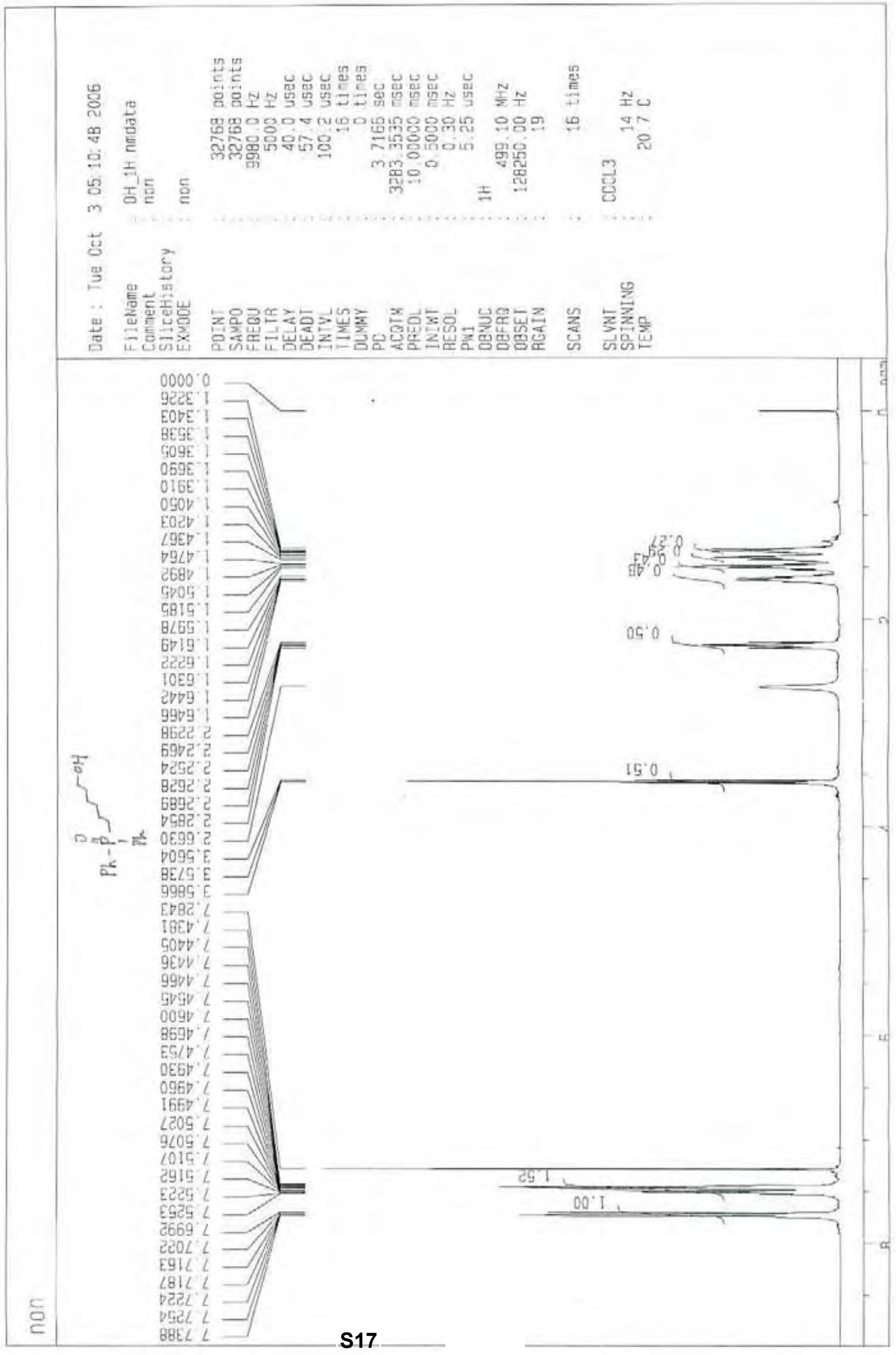




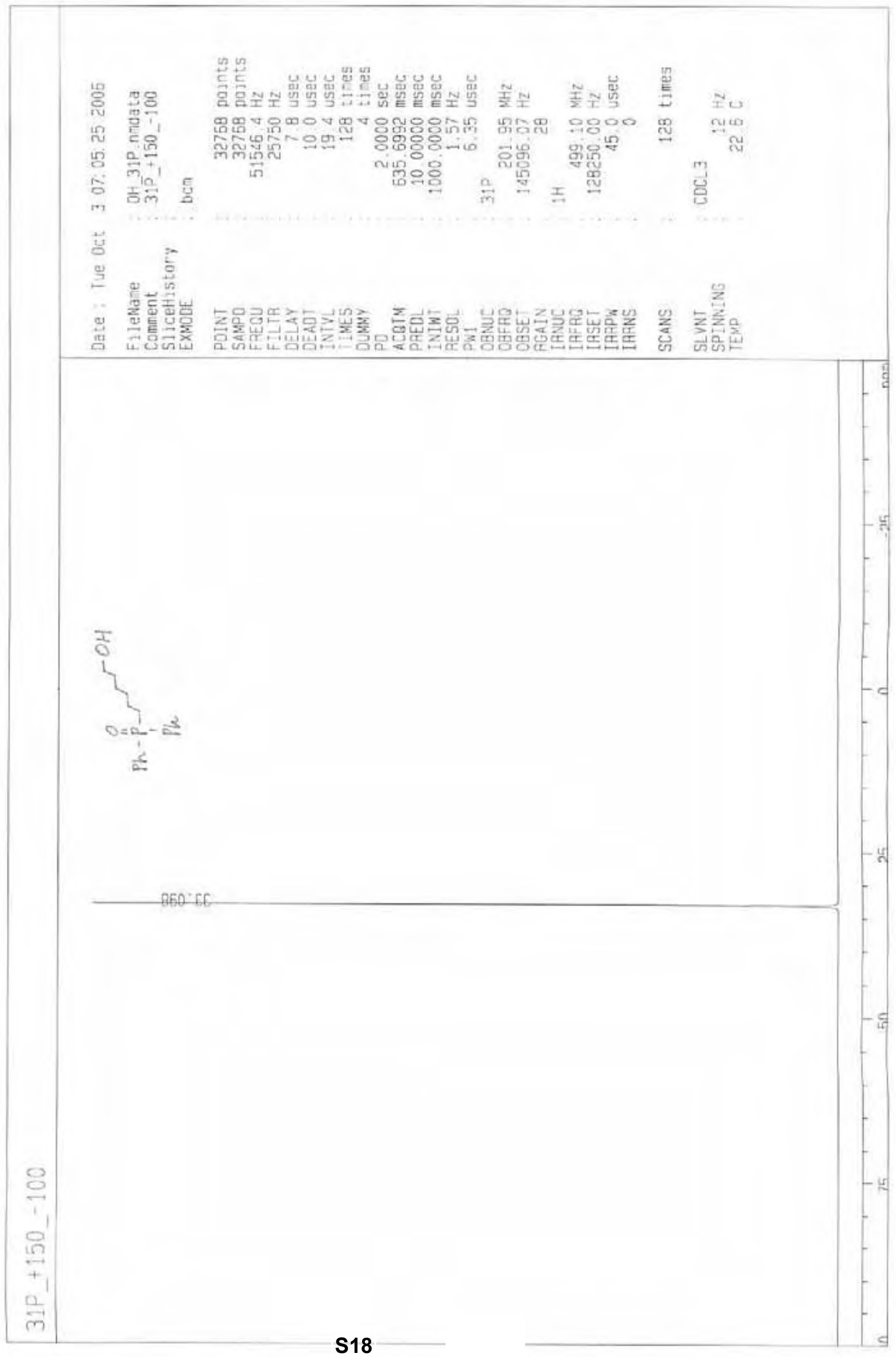




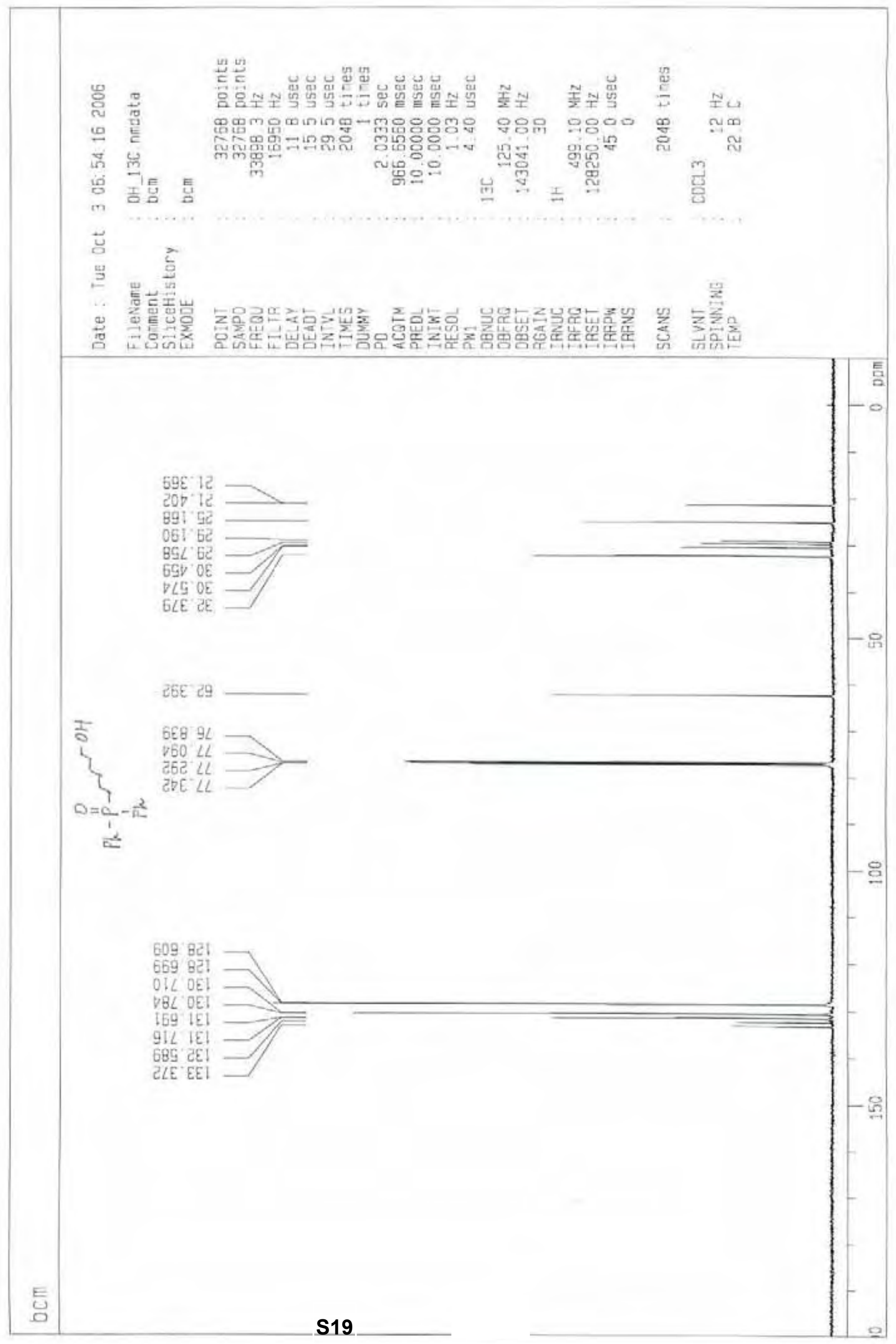




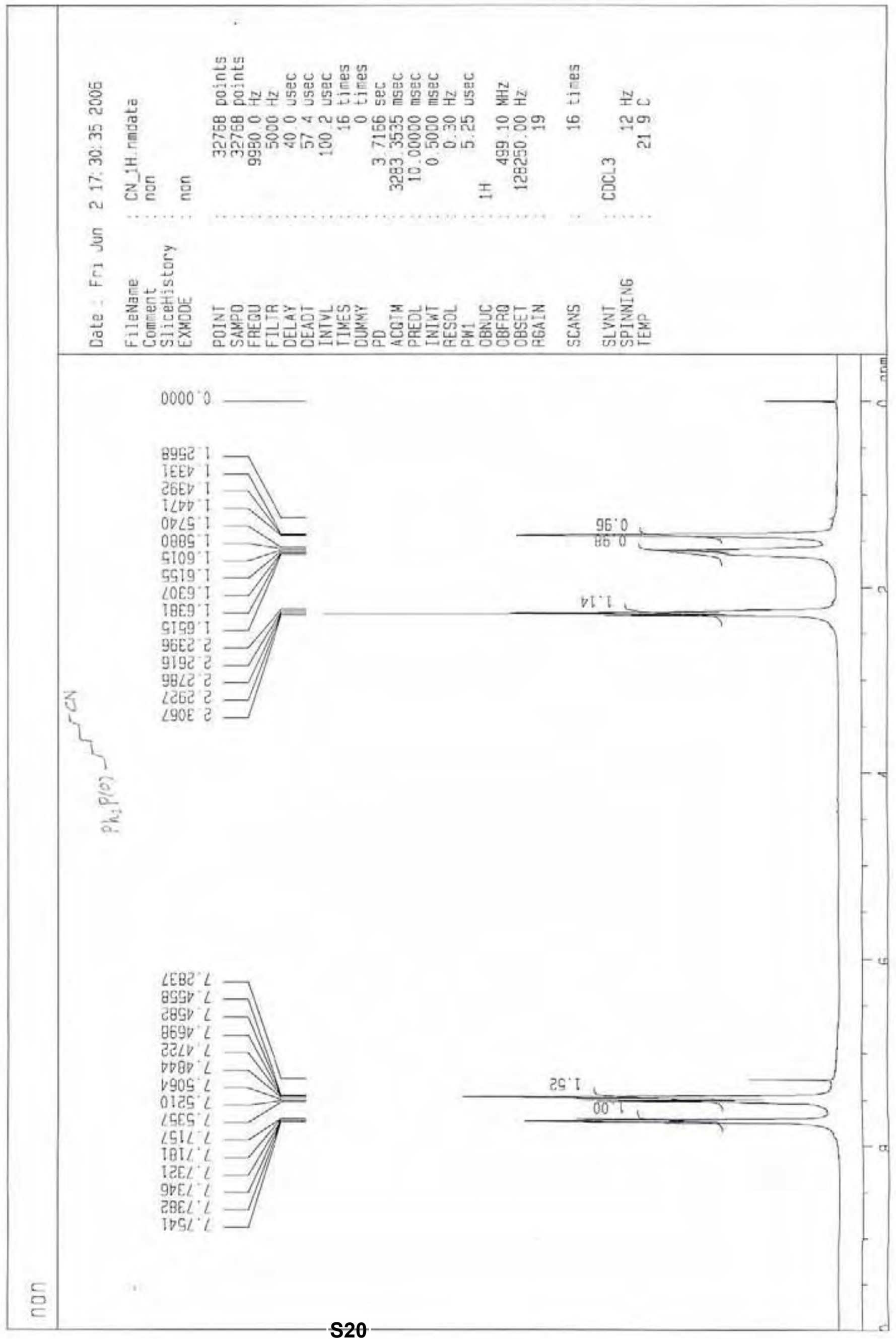




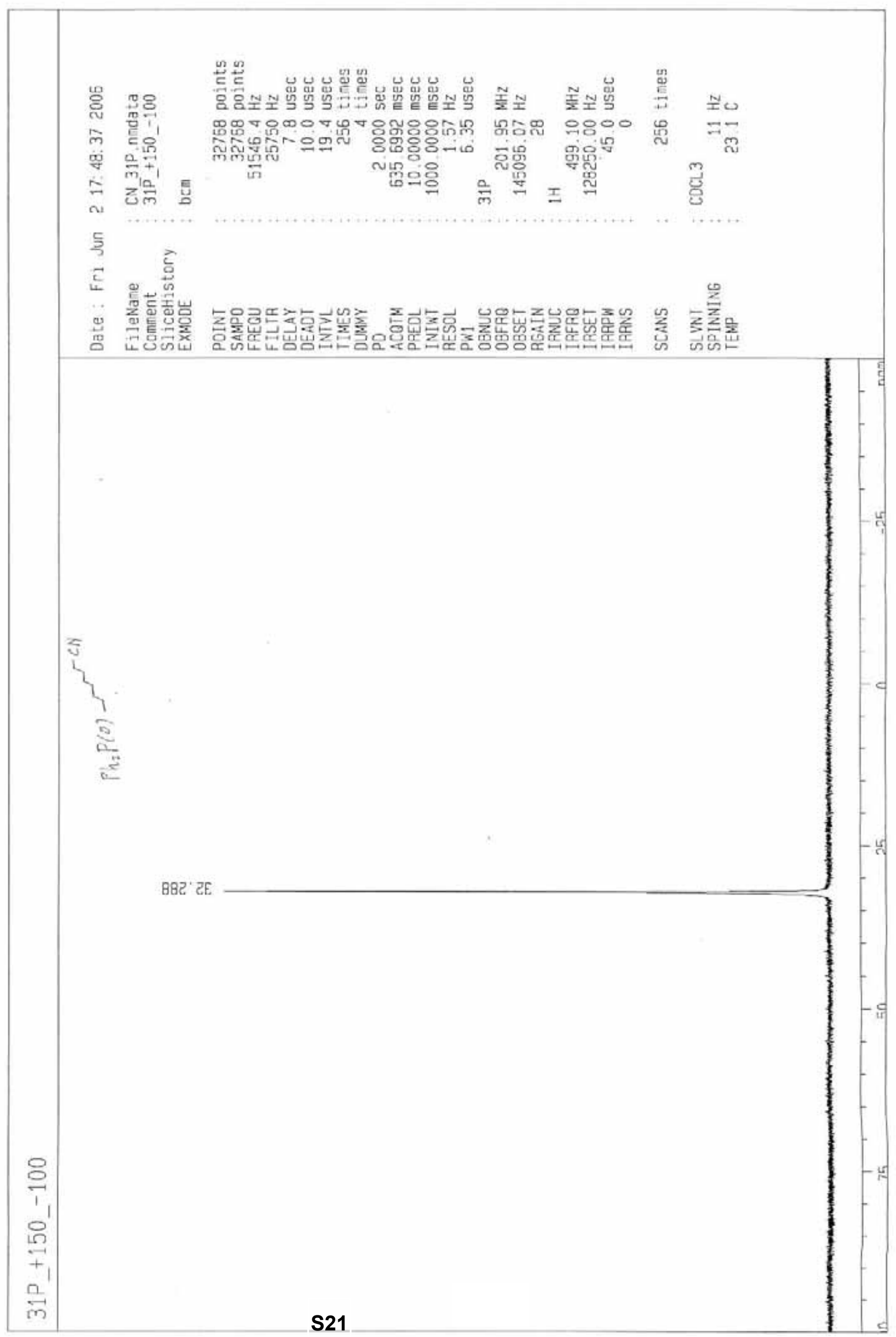









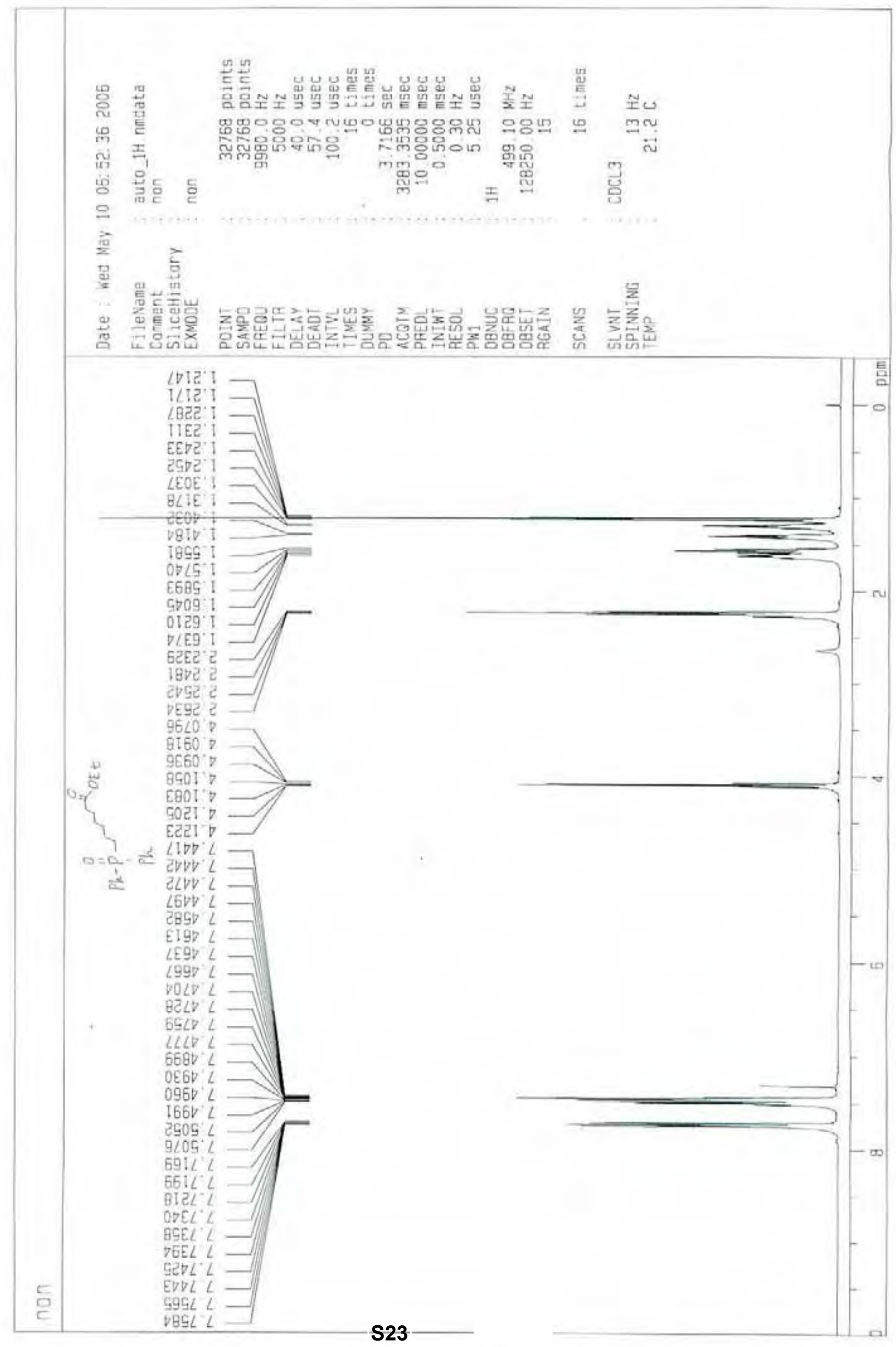




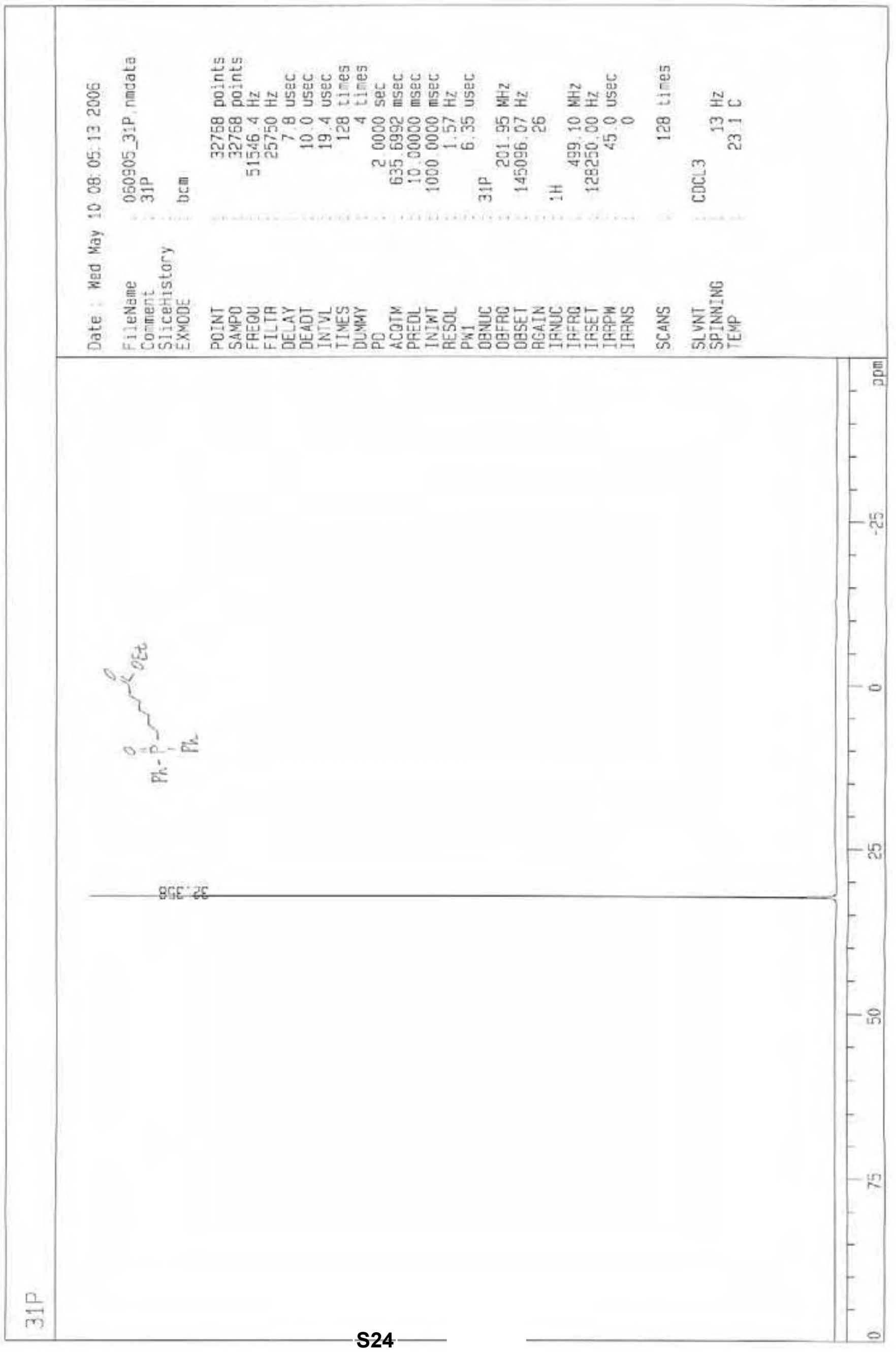









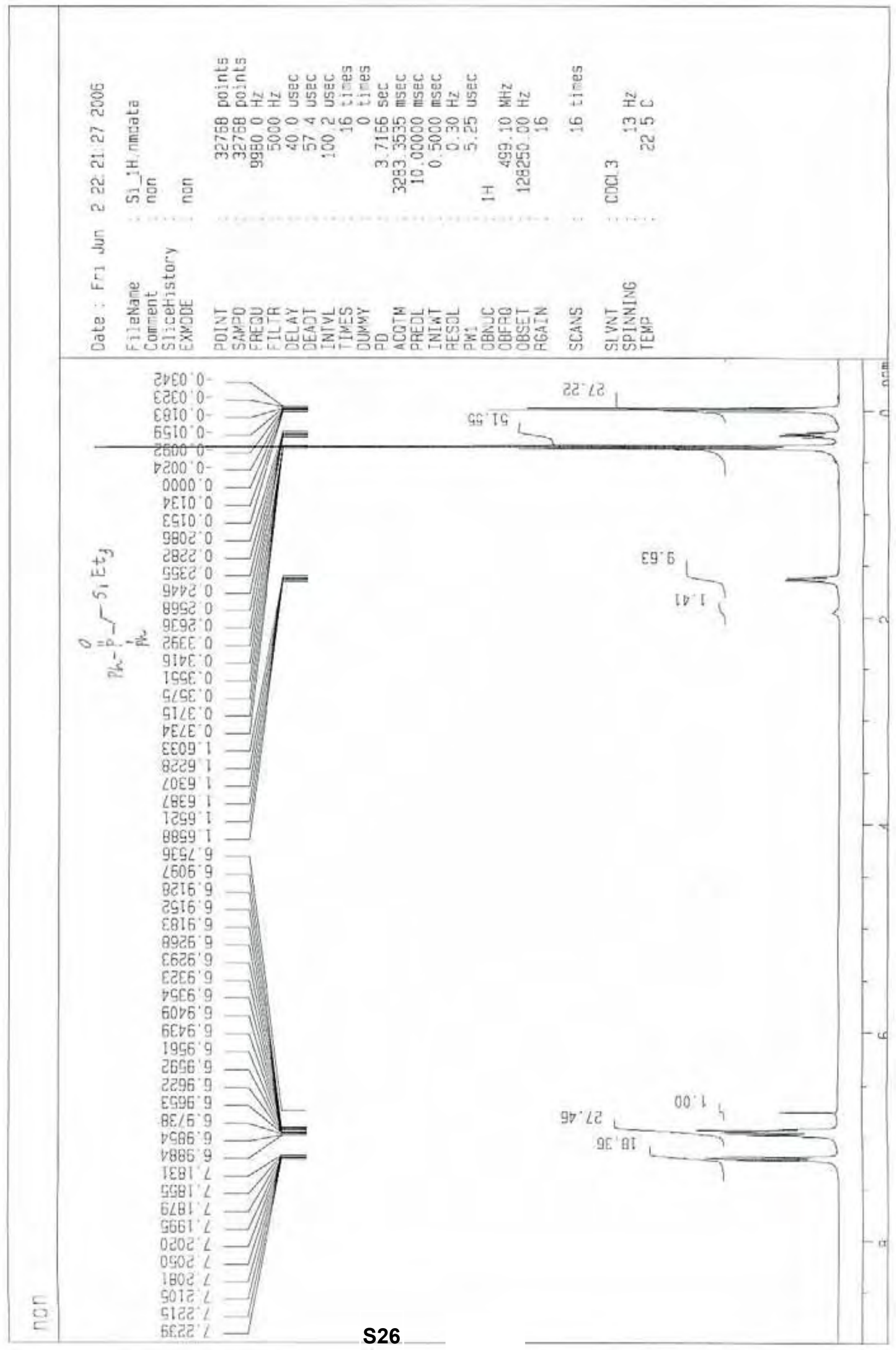




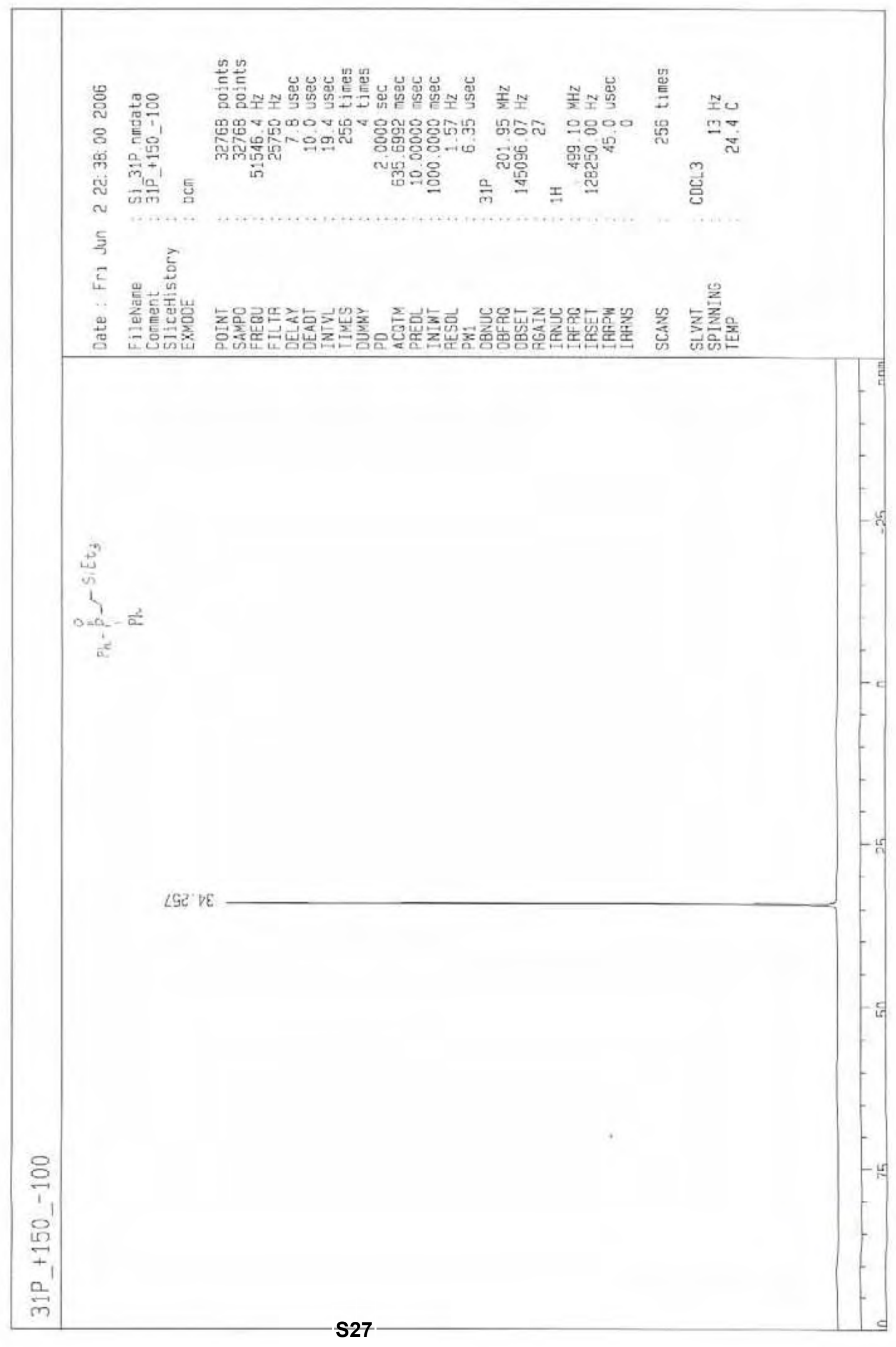




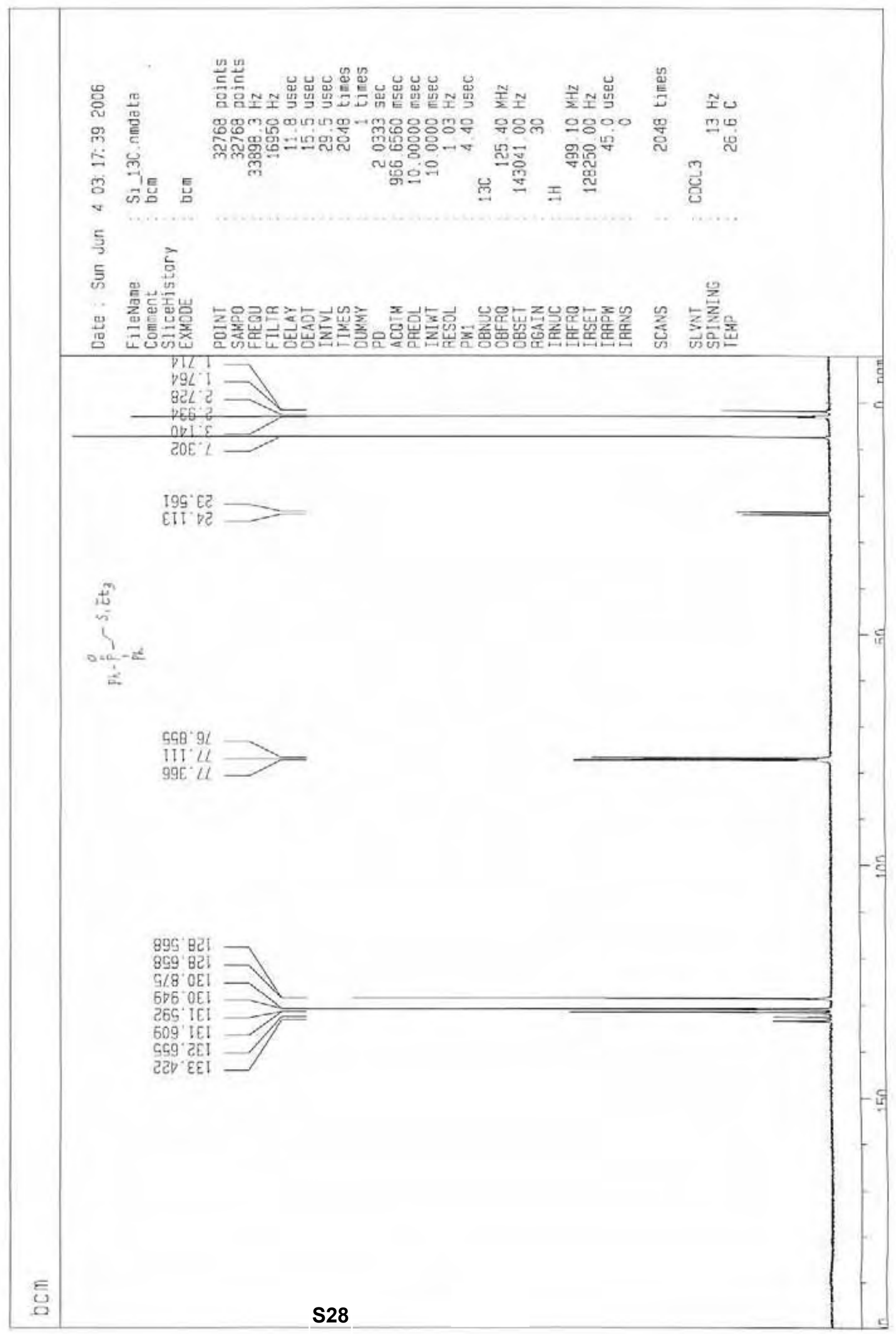




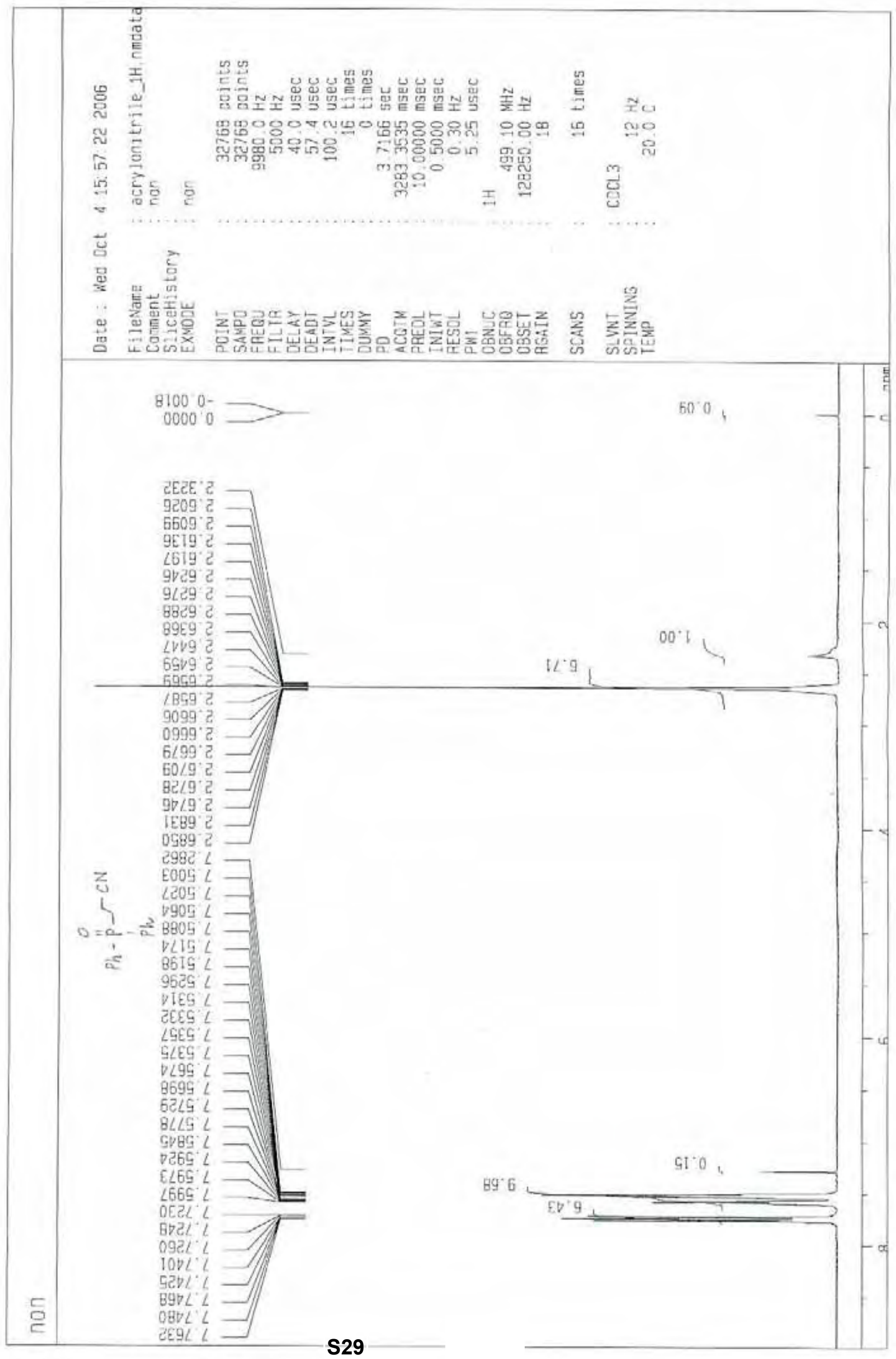




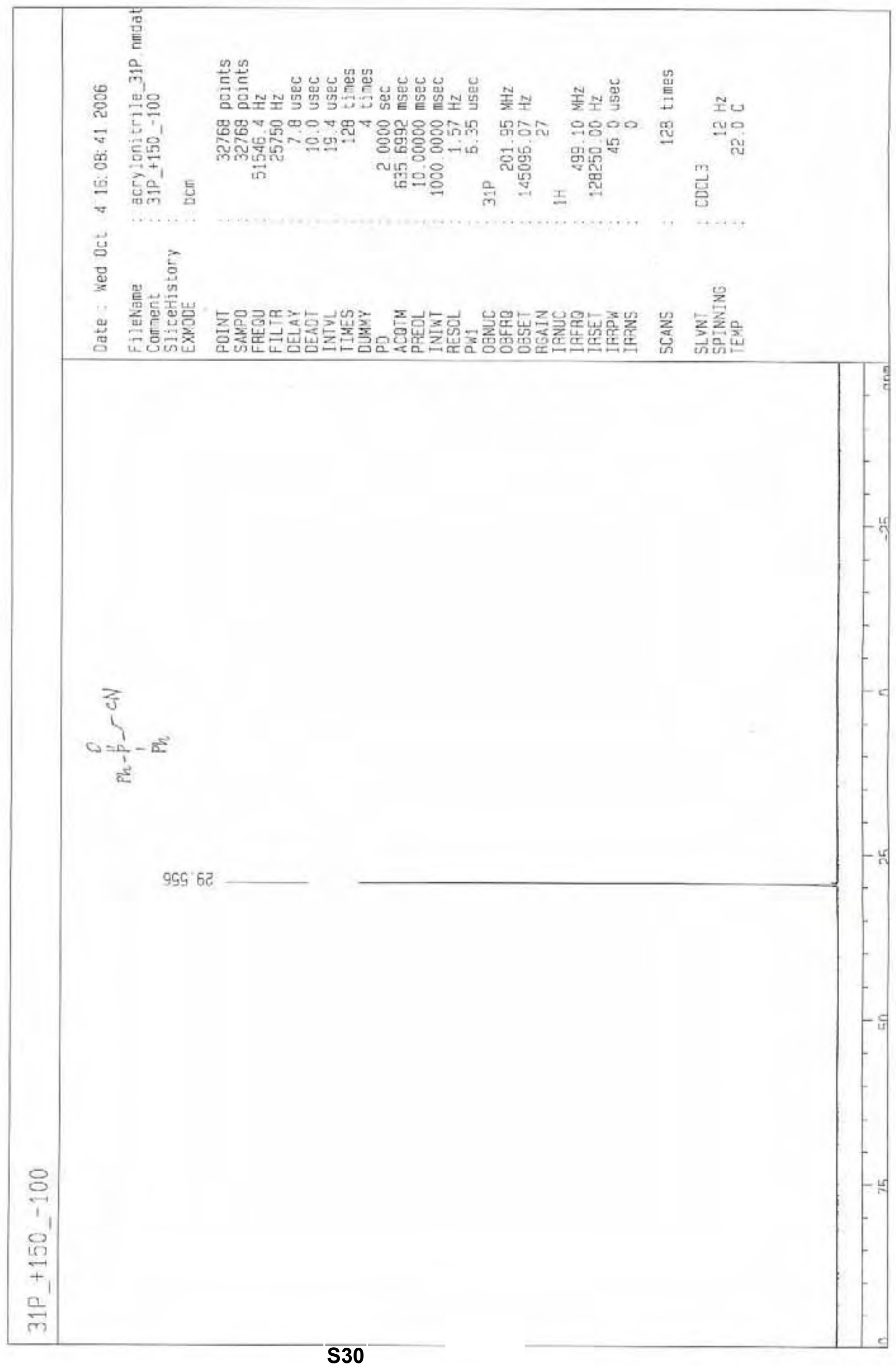

